\title{
Mutations in Rv2983 as a novel determinant of resistance to nitroimidazole drugs in Mycobacterium tuberculosis
}

Dalin Rifat ${ }^{1}$, Si-Yang Li ${ }^{1}$, Thomas loerger ${ }^{2}$, Jean-Philippe Lanoix ${ }^{3}$, Jin Lee ${ }^{1}$, Ghader Bashiri ${ }^{4}$, James Sacchettini ${ }^{5}$ and Eric Nuermberger ${ }^{1, *}$

${ }^{1}$ Center for Tuberculosis Research, Johns Hopkins University School of Medicine, 1550

Orleans Street, Baltimore, MD 21287, USA

${ }^{2}$ Department of Computer Science, Texas A\&M University, College Station, TX, 77843, USA

${ }^{3}$ Department of Infectious Diseases, Amiens University Hospital, Amiens, France

${ }^{4}$ Laboratory of Structural Biology and Maurice Wilkins Center for Molecular Biodiscovery, School of Biological Sciences, University of Auckland, Auckland 1010, New Zealand

${ }^{5}$ Department of Biochemistry and Biophysics, Texas A\&M University, College Station, TX, 77843, USA

*Corresponding author. Email: enuermb@jhmi.edu Tel: 410-502-7683

\section{SIGNIFICANCE}

Nitroimidazole pro-drugs represent a promising new class of anti-tuberculosis drugs. Reliable methods to assure nitroimidazole susceptibility are critical to assure their optimal use. Yet, the spectrum of nitroimidazole resistance mutations remains incompletely characterized. Using 161 pretomanid-resistant Mycobacterium tuberculosis isolates selected in pretomanid-treated mice, we discovered a novel resistance determinant, Rv2983, required for cofactor $F_{420}$ biosynthesis and characterized the remarkable diversity of mutations in this and 5 other genes involved in 
nitroimidazole activation. We show that $\mathrm{F}_{420} \mathrm{H}_{2}$-deficient nitroimidazole-resistant mutants are hypersusceptible to the selective decontaminant malachite green used in solid media to isolate mycobacteria and may evade detection on such media. These results have important implications for development and clinical use of genotypic and phenotypic methods for nitroimidazole susceptibility testing.

\section{ABSTRACT}

Delamanid represents one of two novel antimicrobial classes approved to treat tuberculosis in over 40 years. Pretomanid is another promising nitroimidazole pro-drug in clinical development. Characterization of the full spectrum of mutations conferring resistance to nitroimidazoles and their related phenotypes in Mycobacterium tuberculosis will inform development of suitable genotypic and phenotypic drug susceptibility tests. Here, we used a range of pretomanid doses to select pretomanid-resistant mutants in two pathologically distinct murine TB models. The frequency of spontaneous pretomanid resistance mutations was approximately $10^{-5} \mathrm{CFU}$. Pretomanid demonstrated dose-dependent bactericidal activity and selective amplification of resistant mutants. Whole genome sequencing of 161 resistant isolates from 47 mice revealed 99 unique mutations, $90 \%$ of which were found in 1 of 5 genes previously associated with nitroimidazole activation and resistance. The remaining $10 \%$ harbored isolated mutations in Rv2983. Complementing an Rv2983 mutant with a wild-type copy of Rv2983 restored wild-type susceptibility to pretomanid and delamanid, confirming that loss of Rv2983 function causes nitroimidazole resistance. By quantifying $\mathrm{F}_{420}$ and its precursor $\mathrm{Fo}$ in Mycobacterium smegmatis overexpressing Rv2983 and an M. tuberculosis Rv2983 mutant, we provide evidence that Rv2983 is necessary for $F_{420}$ biosynthesis and nitroimidazole activation, perhaps as the guanylyltransferase CofC. $\mathrm{F}_{420} \mathrm{H}_{2}$-deficient mutants displayed hypersusceptibility to malachite green (MG), a selective decontaminant present in solid media used to isolate and propagate mycobacteria from clinical samples. The wide diversity of mutations causing high-level 
pretomanid resistance and MG hypersusceptibility of most mutants poses significant challenges to clinical detection of nitroimidazole resistance using either genotypic or phenotypic methods.

\section{INTRODUCTION}

Despite decades of efforts to end the global tuberculosis (TB) epidemic, Mycobacterium tuberculosis is the leading killer among infectious agents plaguing mankind (1). The emergence and spread of multidrug-resistant (MDR) and extensively drug-resistant (XDR) M. tuberculosis makes the eradication effort much more difficult because treatment requires administration of more toxic and less effective second- and third-line drugs for up to 2 years $(1,2)$. Delamanid and pretomanid are promising new bicyclic 4-nitroimidazole drugs that have shown potential in preclinical and clinical studies to shorten and simplify the treatment of TB, including drug-resistant forms (3-9). Delamanid received conditional approval by the European Medicines Agency (EMA) to treat MDR-TB in 2014 (10) and pretomanid is currently being evaluated in Phase 2/3 clinical trials (ClinicalTrials.gov Identifiers: NCT03338621, NCT02589782, NCT02333799, NCT03086486). Particularly notable is a novel regimen comprised of bedaquiline, pretomanid and linezolid that may represent a highly efficacious oral, short-course regimen for treatment of MDR/XDR-TB (4)(ClinicalTrials.gov Identifiers: NCT02333799, NCT03086486).

Two non-exclusive mechanisms of action have been described for these bicyclic 4-nitroimidazole drugs: inhibition of cell wall biosynthesis through inhibition of mycolic acid synthesis and respiratory poisoning through release of nitric oxide during bacterial drug metabolism $(11,12)$. Pretomanid and delamanid are prodrugs that require bioreductive activation of an aromatic nitro group by the 8-hydroxy-5-deazaflavin (coenzyme $\mathrm{F}_{420}$ )-dependent nitroreductase $\mathrm{Ddn}$ in order to exert bactericidal activity (13). The reaction involves the transfer of two electrons from the reduced form of $\mathrm{F}_{420}\left(\mathrm{~F}_{420} \mathrm{H}_{2}\right)$ produced by an $\mathrm{F}_{420}$-dependent glucose-6-phosphate dehydrogenase (Fgd1) $(12,14)$. Therefore, $F_{420}$ biosynthesis and reduction are essential for the activation of delamanid, pretomanid and other nitroimidazole prodrugs. Three genes are identified as essential for $F_{420}$ 
biosynthesis in M. tuberculosis complex $(15,16)$. fbiC encodes a 7,8-didemethyl-8-hydroxy-5deazariboflavin (Fo) synthase that catalyzes the condensation of 5-amino-6-ribitylamino-2,4 $(1 \mathrm{H}$, $3 H$ )-pyrimidinedione and tyrosine to form the $F_{420}$ precursor $F o(17,18)$. fbiA encodes a transferase that is believed to catalyze the transfer of a phospholactyl moiety to Fo to generate $\mathrm{F}_{420} \mathrm{O}$, while $\mathrm{fbiB}$ encodes a $\mathrm{F}_{420}-0: \mathrm{Y}-\mathrm{L}$-glutamyl ligase that catalyzes the sequential addition of a variable number of glutamate residues to $F_{420}-0$ to yield coenzyme $F_{420}-5$ or -6 in mycobacteria (12). In the methanogen Methanocaldococcus jannaschii, a guanylyltransferase termed CofC is believed to generate an intermediate (L-lactyl-2-diphospho-5'-guanosine,-LPPG) in the $F_{420}$ biosynthesis pathway (19). A homologous enzyme, MSMEG_2392, is shown to be necessary for $\mathrm{F}_{420}$ synthesis in Mycobacterium smegmatis through transposon mutagenesis studies (20). An ortholog, Rv2983, is present in M. tuberculosis. However, the role of MSMEG-2392 and Rv2983 in $\mathrm{F}_{420}$ biosynthesis has remained unexplored.

Loss-of-function mutations in $d d n, f g d 1$ and $f b i A-C$ causing delamanid and pretomanid resistance are readily selected in vitro in $M$. tuberculosis complex $(16,18,21-23)$. However, the genetic spectrum of mutations emerging during in vivo selection has not been characterized. In order to study bacterial genetic, host and pharmacological factors associated with emergence of nitroimidazole resistance in vivo, we selected pretomanid-resistant mutants using a wide range of pretomanid doses in two mouse models of TB and characterized them by whole genome sequencing (WGS). Because the lungs of TB patients feature a heterogeneous array of lesion types associated with diversified immune responses and drug penetration $(24,25)$, we used both $\mathrm{C} 3 \mathrm{HeB} / \mathrm{FeJ}$ mice, which develop caseating lung lesions in response to $M$. tuberculosis infection, and BALB/c mice, which do not, to investigate the impact of these caseating lesions and their associated micro-environments on mutant selection. In the present study, we found that pretomanid-resistant mutants were readily selected by monotherapy in both mouse strains. While the majority of resistant isolates harbored isolated mutations in genes previously associated with 
nitroimidazole resistance, all the resistant isolates lacking such mutations had mutations in Rv2983. We went on to confirm that loss-of-function mutations in Rv2983 cause high-level pretomanid and delamanid resistance through disruption of $F_{420}$ biosynthesis, supporting the hypothesis that Rv2983 plays a role similar to cofC in M. tuberculosis. Furthermore, $\mathrm{F}_{420} \mathrm{H}_{2-}$ deficient nitroimidazole-resistant M. tuberculosis mutants, including Rv2983 mutants, were found to be hypersensitive to malachite green (MG), an organic compound used as a selective decontaminant in solid media for culturing M. tuberculosis, which may have important implications for their detection in clinical samples.

\section{MATERIALS AND METHODS}

Bacterial strains, media, antimicrobials and reagents. Wild type $M$. tuberculosis H37Rv (ATCC 27294) was mouse-passaged, frozen in aliquots and used in all the experiments. The wild type M. smegmatis strain $\mathrm{mc}^{2} 155$ was obtained from the stock in the lab. Unless stated otherwise, Middlebrook $7 \mathrm{H} 9$ medium (Difco, BD) supplemented with $10 \%$ oleic acid-albumin-dextrosecatalase (OADC) complex (BD), 0.5\% glycerol and 0.05\% Tween 80 (Sigma-Aldrich) (7H9 broth) was used for cultivation. Middlebrook 7H10 agar and selective 7H11 agar (Difco, BD), prepared from powder and containing 10\% OADC and $0.5 \%$ glycerol, were used for comparison of strain recovery on commercially available agar plates. Lowenstein Jensen (LJ) slants were purchased from BD. Pretomanid and delamanid were kindly provided by the Global Alliance for TB Drug Development (New York, NY).

Mouse infection models and pretomanid treatment. All animal procedures were approved by the Animal Care and Use Committee of Johns Hopkins University. Aerosol infections were performed using the Inhalation Exposure System (Glas-col Inc., Terre Haute, IN), as previously described (26). Briefly, 6-week-old female BALB/c mice (Charles River, Wilmington, MA) and 
C3HeB/FeJ mice (Jackson Laboratories Bar Harbor, ME) were infected with a log phase culture of $M$. tuberculosis that was grown in $7 \mathrm{H} 9$ broth to $0 . D \cdot 600_{\mathrm{nm}}=1.0$ and then diluted in the same medium prior to infection to deliver 50-100 CFU to the lungs. Pretomanid was formulated for oral administration as previously described (27). Beginning 8 weeks after aerosol infection, mice were randomly allocated into groups and treated once daily (5 days per week) for up to 8 weeks with pretomanid at doses of $10,30,100,300$ and $1000 \mathrm{mg} / \mathrm{kg}$. Untreated mice were sacrificed on the day after aerosol infection and on the day of treatment initiation to determine the number of CFU implanted in the lungs and pretreatment CFU counts, respectively. Additional mice were sacrificed after 3 and 8 weeks of treatment to evaluate the treatment response. Serial 10 -fold dilutions of lung homogenates were plated on $7 \mathrm{H} 11$ agar. Week 8 samples including those from untreated mice were also plated in parallel on $7 \mathrm{H} 11$ plates containing $0.25,1$ and $10 \mu \mathrm{g} / \mathrm{ml}$ of pretomanid to quantify the resistant CFU. Plates were incubated at $37^{\circ} \mathrm{C}$ for 28 days before final CFU counts were determined.

Whole genome sequencing. For each mouse lung that yielded growth on pretomanid-containing plates, individual colonies and, for a subset of mice, pools of up to 15 colonies, were randomly selected from pretomanid-containing plates and sub-cultured in $7 \mathrm{H} 9$ broth prior to extraction of genomic DNA using the cetyltrimethylammonium bromide (CTAB) protocol (28) and vortexing (Genegate, Inc.). 2-3 $\mu$ g of genomic DNA was sheared by a nebulizer to generate DNA fragments. The DNA library was prepared using a genomic DNA sample preparation kit (Illumina, Inc.), in which adapter-ligated DNA fragments were 250-350 bp in length, and carried out on an Illumina Genome Analyzer II (Illumina, Inc). The sequencer was operated in paired-end mode to collect pairs of reads of 51-bp from opposite ends of each fragment. Image analysis and base-calling were done by using the Illumina GA Pipeline software (v0.3). The reads that were generated for each strain were aligned to the reference genome of $M$. tuberculosis H37Rv (29). Based on alignment to the corresponding region in the reference genome, single nucleotide polymorphism 
(SNP), insertion and deletion were identified on the genome of resistant strains by using a contigbuilding algorithm to construct a local $\sim 200$ bp sequence spanning the site of mutagenesis (30). Distribution of mutation type and mutation frequency in genes involved in nitroimidazole resistance was calculated by counting the total number of unique mutations isolated from each mouse in the same treatment group.

Complementation of an Rv2983 mutation. A 1,044-bp DNA fragment containing the open reading frame (ORF) of the wild type Rv2983 gene, including 340 bp of 5'-flanking sequence and 59 bp of 3'-flanking sequence, was PCR-amplified from M. tuberculosis H37Rv genomic DNA using primers Rv2983-1F and Rv2983-1R (Table S1). The Rv2983 PCR product was ligated into Xbal-digested E. coli-mycobacterium shuttle vector pMH94 (31) using NE builder HiFi DNA assembly kit (NE Biolabs) to generate the recombinant pMH94-RV2983 vector. Similarly, a 388bp DNA fragment containing the $h s p 60$ promoter and a 645-bp DNA fragment of Rv2983 open reading frame were amplified from $M$. tuberculosis H37Rv genomic DNA using primer sets hsp60F and $h s p 60-R$ and Rv2983-2F and Rv2983-2R, respectively (Table S1), and ligated into Xbaldigested E. coli-mycobacterium shuttle vector pMH94 to yield pMH94-hsp60-Rv2983. A small amount of ligation reaction was transferred into E. coli competent cells, followed by DNA sequencing of the inserts in the corresponding recombinants. The recombinants pMH94-RV2983 and pMH94-hsp60-Rv2983 were electroporated into competent cells of Rv2983 mutant strain BA_101 (B101), harboring an A198P substitution, to enable selection of complemented candidates B101pRv2983 and B101phsp60-Rv2983 on 7H10 agar containing $25 \mu \mathrm{g} / \mathrm{ml}$ of kanamycin. To confirm the complementation genetically, Southern blotting was performed using a digoxigenin (DIG) DNA labeling and detection kit according to the manufacturer's protocol (Sigma). Briefly, a 448-bp Rv2983 probe was generated by addition of DIG-dUTP (Sigma) to PCR reactions containing primer pairs Rv2983-3F and Rv2983-3R (Table S1). Acc65I-digested (NE biolabs) genomic DNA of the wild type, the B101 mutant and the B101pRv2983 and B101phsp60- 
Rv2983 complemented strains was separated on agarose gel and transferred onto positivelycharged nylon-membrane (GE). After pre-hybridization, the membrane was hybridized with the DIG-labeled Rv2983 probe at $68^{\circ} \mathrm{C}$ overnight, followed by addition of anti-DIG alkaline phosphatase conjugate. After stringent washes, the membrane was incubated with the chemiluminescence substrate disodium 3-(4-methoxyspiro $\quad$ 1,2-dioxetane-3,2(5'chloro)tricycloecan\}-4-yl)phenyl phosphate (CSPD) and exposed on X-ray film in a dark room prior to development using a developer (AFP imaging)(32).

MIC determination. Log-phase cultures were diluted to achieve a bacterial density of approximately $10^{5} \mathrm{CFU} / \mathrm{ml}$ in conical tubes containing $7 \mathrm{H} 9$ broth without Tween 80 . Serial 10 -fold dilutions were plated on $7 \mathrm{H} 11$ agar containing stepwise 2-fold increasing pretomanid concentrations ranging from 0.015 to $64 \mu \mathrm{g} / \mathrm{ml}$ or delamanid concentrations from 0.001 to 1.024 $\mu \mathrm{g} / \mathrm{ml}$. Drugs were initially dissolved in dimethylsulfoxide (DMSO) (Sigma) prior to further dilution in $7 \mathrm{H} 9$ broth or $7 \mathrm{H} 11$ agar. Cultures were incubated at $37^{\circ} \mathrm{C}$ for 14 days or 28 days after plating. MIC was defined as the lowest drug concentration that inhibited visible $M$. tuberculosis growth in conical tubes or that inhibited $99 \%$ of CFU growth on pretomanid-containing plates $(33,34)$. The experiments were repeated twice.

\section{Construction of recombinants overexpressing Rv2983, with or without fbiC, in $M$.}

smegmatis. A 645-bp DNA fragment containing the Rv2983 ORF was PCR-amplified from M. tuberculosis H37Rv genomic DNA using primers Rv2983-4F and Rv2983-4R (Table S1). The amplified PCR product was ligated into the Ndel- and Pacl-digested E. coli-mycobacterium shuttle vector pYUBDuet (35) using NE builder HiFi DNA assembly kit (NE Biolabs) and then transferred into Turbo-competent E. coli cells (NE Biolabs) prior to plating on LB agar plates containing $100 \mu \mathrm{g} / \mathrm{ml}$ of hygromycin B for selection of recombinants. The Rv2983 PCR product was also similarly ligated into the same Ndel- and Pacl-digested pYUBDuet vector harboring 
fbiC (termed pfbiC) (35) to overexpress both Rv2983 and fbiC. After confirmation by restriction digestion and DNA sequencing, the constructs were electroporated into competent $M$. smegmatis cells prior to selecting recombinants on $7 \mathrm{H} 10$ agar plates containing $100 \mu \mathrm{g} / \mathrm{ml}$ of hygromycin B. PCR amplification was used to confirm the inserts on the M. smegmatis genome. pYUBDuet and pYUBDuet harboring fbiA, fbiB and fbiC (termed pfbiABC) (35) were also transferred into competent $M$. smegmatis cells to serve as controls.

Measurement of Fo and $\mathbf{F}_{420}$. Extraction of $F_{0}$ and $F_{420}$ was performed in $M$. smegmatis and $M$. tuberculosis strains according to a previous study (35), with minor modifications. Briefly, $M$. smegmatis strains harboring different constructs and pYUBDuet were grown in $7 \mathrm{H} 9$ broth in a shaker to mid-log phase (O.D.600nm $=0.7-1.0)$, followed by induction using $1 \mathrm{mM}$ isopropyl $\beta-D-1$ thiogalactopyranoside (IPTG) for 6 and 26 hours. After centrifugation for $15 \mathrm{~min}$ at $16000 \mathrm{xg}$, the supernatants were removed for detection of Fo, which is principally found in culture supernatant whereas $F_{420}$ with 5 or 6 glutamate residues is largely retained inside cells $(15,35,36)$. The cell pellets were washed with $25 \mathrm{mM}$ sodium phosphate buffer $(\mathrm{pH} 7.0)$ and re-suspended at 100 $\mathrm{mg} / \mathrm{mL}$ in the same buffer, then autoclaved at $121^{\circ} \mathrm{C}$ for $15 \mathrm{~min}$. After centrifugation at $16000 \times \mathrm{g}$ for $15 \mathrm{~min}$ at $4^{\circ} \mathrm{C}$, the cell extracts were harvested for detection of $\mathrm{F}_{420}(35)$. Fluorescence of the supernatant and cell extracts was measured using an excitation wavelength of $410 \mathrm{~nm}$ and an emission wavelength of $465 \mathrm{~nm}$. Fluorescent signals of Fo were normalized using the O.D. at $600 \mathrm{~nm}$. The small portion of Fo (1-7\%) retained inside cells was ignored when quantifying $\mathrm{F}_{420}$ in cell extracts (37). Relative fluorescent signals were calculated in M. smegmatis harboring each of recombinants relative to pYUBDuet alone. Similarly, cell extracts and supernatant were extracted from M. tuberculosis strains grown in $7 \mathrm{H} 9$ broth for 6 days at initial O.D.600nm of 0.1. Relative fluorescent signals of $\mathrm{F}_{420}$ and Fo were calculated using cell extracts and supernatant relative to $25 \mathrm{mM}$ phosphate buffer and $7 \mathrm{H} 9$ broth, respectively. M. smegmatis harboring pYUBDuet-fbiABC 
was used as a positive signal control for $\mathrm{Fo}$ and $\mathrm{F}_{420}$ due to their commercial unavailability (35). The experiment was repeated twice.

Quantification of gene expression in M. tuberculosis. 6-day-old M. tuberculosis strains grown in $7 \mathrm{H} 9$ broth as described above were sub-cultured in fresh $7 \mathrm{H} 9$ at O.D.600nm $=0.05$ followed by incubation at $37^{\circ} \mathrm{C}$ in a shaker for 2 and 4 days. Bacterial pellets were collected by centrifugation at $3500 \mathrm{rpm}$ at $4^{\circ} \mathrm{C}$ for purification of total RNA using Trizol (Thermo Fisher Scientific) according to the manufacturer's protocol followed by removal of DNA contamination with Turbo DNAse (Ambion). Following cDNA synthesis with random hexamers and oligo(dT)20 primer and superscript III reverse transcriptase (Invitrogen), quantitative PCR was performed to measure gene expression of M. tuberculosis using SYBR Green PCR master mix (Thermo scientific) and StepOne ${ }^{\mathrm{TM}}$ system (Applied biosystems) with primer sets listed in Table S1. The cycle threshold value $\left(\mathrm{C}_{\mathrm{T}}\right)$ measured for each gene was normalized to that of the housekeeping gene $\operatorname{sig} A\left(\Delta \mathrm{C}_{\mathrm{T}}\right)$ amplified by the primers sigA-F and $\operatorname{sig} A-\mathrm{R}$ (Table $\mathrm{S} 1$ ). $\Delta \Delta \mathrm{C}_{\mathrm{T}}$ was calculated in each of pretomanid-resistant strains relative to the wild-type H37Rv prior to calculation of the fold-change in gene expression $\left(2^{\wedge}-\Delta \Delta C_{T}\right)(38)$. All samples were prepared in duplicate. PCR was performed from an equal amount of cDNA samples synthesized with oligo(dT)20 with primers fbiC-5-7_F and fbiC-5-7_R (Table S1) using the $Q_{5}$ High-fidelity PCR kit (New England Biolabs) and C1000 Thermal cycler (Biorad). The PCR product was examined by electrophoresis on a $1 \%$ agarose gel.

Malachite green susceptibility testing. $7 \mathrm{H} 9$ media supplemented with $10 \%$ OADC, $0.5 \%$ glycerol, $1.5 \%$ Bacto $^{\mathrm{TM}}$ Agar (BD) and malachite green (MG) oxalate (Alfa Aesar) was used to prepare solid $7 \mathrm{H} 9$ media with differing MG concentrations. M. tuberculosis strains were grown to mid-log phase and diluted to $\mathrm{OD}_{600 \mathrm{~nm}}=0.1$ in $7 \mathrm{H} 9$ broth before serial 10 -fold dilutions were plated in 100 or $500 \mu \mathrm{l}$ aliquots on $7 \mathrm{H} 9$ agar containing MG concentrations of $0,0.1,0.3,1,3,10,30$, 
$100,300,1000 \mu \mathrm{g} / \mathrm{ml}$ or $0,3,6,12 \mu \mathrm{g} / \mathrm{ml}$. CFU were counted after 28,35 and 49 days of incubation. The same cultures were also plated on $7 \mathrm{H} 10$ and $7 \mathrm{H} 11$ agar plates and LJ slants. Serially diluted cultures were inoculated onto LJ slants using calibrated disposable inoculating loops (10 $\mu$ l per loop, BD) as one loop per LJ slant. Plates were incubated at $37^{\circ} \mathrm{C}$ for 21,28 and 35 days for CFU counts. Colony size was observed weekly until day 35, beginning 21 days after plating. The experiment was repeated two times under similar conditions.

Statistical analysis. $\log _{10}$-transformed CFU counts, fold-change values of gene expression and absorbance $\left(A_{410}\right)$ values of fluorescent signals were used to calculate means and standard deviations for each data set. Differences between means were compared by the Student's $t$ test in Microsoft Excel. Differences in mutation frequencies between two mouse models were evaluated by Fisher's exact test in GraphPad Prism 6. A p-value of $<0.05$ was considered statistically significant.

\section{RESULTS}

\section{Spontaneous pretomanid-resistant mutants exist at a relatively high frequency in} infected mice and are selectively amplified by treatment with active doses of pretomanid.

To study the dose-response of pretomanid and explore the genetic spectrum of nitroimidazole resistance selected in vivo, we established chronic $M$. tuberculosis infections in BALB/C and $\mathrm{C} 3 \mathrm{HeB} / \mathrm{FeJ}$ mice and then treated with a range of pretomanid doses for up to 8 weeks. Despite lower CFU counts on the day after infection (W-8) in C3HeB/FeJ mice (1.67 $\log _{10}$ CFU per lung) compared to BALB/c $\left(2.26 \log _{10}\right)(p<0.001)$, higher CFU counts were observed in C3HeB/FeJ mice 8 weeks later on the day treatment started (D0) and after 3 weeks of treatment in almost all groups $(p<0.001-0.05)($ Fig. 1A). Three C3HeB/FeJ mice treated with $1000 \mathrm{mg} / \mathrm{kg}$ required euthanasia during the second week of treatment, prompting a dose reduction from $1000 \mathrm{mg} / \mathrm{kg}$ to $600 \mathrm{mg} / \mathrm{kg}$ in both strains. Nevertheless, a clear pretomanid dose-response relationship was 
observed in both mouse strains after 3 weeks of treatment (Fig. 1A). The three remaining C3HeB/FeJ mice treated with $600 \mathrm{mg} / \mathrm{kg}$ beyond the week 3 time point were euthanized after 5 weeks of treatment due to toxicity. One had no detectable CFU and two had $\leq 2.0 \log _{10} \mathrm{CFU}$ of pretomanid-resistant M. tuberculosis. After 8 weeks of treatment, total CFU counts fell in a dosedependent manner in BALB/c mice before a plateau was reached at doses $\geq 300 \mathrm{mg} / \mathrm{kg}$, where resistant CFU were higher and replaced the susceptible CFU $(p<0.05)$ (Fig. 1B). Spontaneous pretomanid-resistant CFU comprised approximately $10^{-5}$ of the total CFU in the absence of drug pressure in untreated BALB/c mice and the proportion of the total CFU that was comprised of pretomanid-resistant CFU increased with dose up to the $300 \mathrm{mg} / \mathrm{kg}$ dose group. Dose-dependent bactericidal activity was also observed in $\mathrm{C} 3 \mathrm{HeB} / \mathrm{FeJ}$ mice (Fig. 1C). However, selective amplification of pretomanid-resistant mutants was more extensive and occurred at lower doses than in BALB/c mice (Fig. 1B and 1C). We were not able to measure the spontaneous frequency of resistant mutants in untreated $\mathrm{C} 3 \mathrm{HeB} / \mathrm{FeJ}$ mice because they succumbed to infection prior to week 8. Pretomanid-resistant CFU replaced susceptible CFU in C3HeB/FeJ mice receiving doses as low as $30 \mathrm{mg} / \mathrm{kg}$ and pretomanid-resistant CFU counts were roughly 10 times higher in $\mathrm{C} 3 \mathrm{HeB} / \mathrm{FeJ}$ mice compared to BALB/c mice (Fig. 1B and C), which indicates greater potential for selective amplification of pretomanid resistance with monotherapy in this strain. Most resistant isolates grew on plates containing $10 \mu \mathrm{g} / \mathrm{ml}$ of pretomanid, but some had fewer CFU on plates containing $10 \mu \mathrm{g} / \mathrm{ml}$ than on those containing $1 \mu \mathrm{g} / \mathrm{ml}$ of pretomanid.

\section{Whole genome sequencing of pretomanid-resistant mutants revealed diverse mutations in Rv2983 or in one of five other genes required for pretomanid activation.}

To characterize mutations associated with pretomanid resistance in vivo, we performed WGS on 136 individual pretomanid-resistant colonies and 25 colony pools picked from 47 individual mice harboring pretomanid-resistant CFU after 8 weeks of treatment (Table S2 and S3). Each individual isolate had an isolated mutation in Rv2983 or one of the 5 genes previously shown to 
be required for pretomanid activation. Overall, 99 unique mutations in these 6 genes were identified from individual and pooled isolates (Table 1 and 2). Except for mutations K9N (fgd1), R322L (fbiC) and Q120P (fbiA), which were shared by two mice each, no two mice harbored the same mutation, which emphasizes the large target size for resistance-conferring mutations. In both $\mathrm{BALB} / \mathrm{c}$ and $\mathrm{C} 3 \mathrm{HeB} / \mathrm{FeJ}$ mice, more than half of the resistant isolates were fbiC mutants (54 and $56 \%$, respectively) (Table 3). For the other five genes, the rank order by mutation frequency was Rv2983 $(15 \%)>$ fbiA $(13 \%)>d d n(9 \%)>f b i B(6 \%)>f g d 1(4 \%)$ in BALB/c mice and fbiA $(18 \%)>d d n(16 \%)>f g d 1$ or Rv2983 $(4 \%)>f b i B(2 \%)$ in C3HeB/FeJ mice. No significant differences in mutation frequencies between BALB/C and $\mathrm{C} 3 \mathrm{HeB} / \mathrm{FeJ}$ mice were observed, although a trend towards more Rv2983 mutations in BALB/c mice (8/54, 15\% of all mutations) compared to $\mathrm{C} 3 \mathrm{HeB} / \mathrm{FeJ}$ mice $(2 / 45,4 \%)$ was detected. The mutations identified in Rv2983 included 8 point mutations resulting in the following amino acid substitutions: R25S, R25G, A68E, A132V, G147C, C152R, Q114R and A198P, as well as an insertion of C after A27 and a deletion of I129 (-ATC) (Tables 1 and 2). The overall frequency distribution of unique mutations was as follows: fbiC $(55 \%, n=54)$, fbiA $(15 \%, \mathrm{n}=15)$, ddn (12\%, $\mathrm{n}=12), \operatorname{Rv} 2983(10 \%, \mathrm{n}=10)$, fgd1 $(4 \%$, $n=4)$, and fbiB $(4 \%, n=4)$ (Fig. 2 and Table S4). There were no clear associations between pretomanid dose and the mutated gene. Mutations in fbiC comprised a higher proportion of those selected in our in vivo study compared to the proportion selected in a previous in vitro study (26\%, $p=0.0001)(22)$. On the other hand, mutations in $d d n(29 \%)$ were more frequent after in vitro selection than in our mouse models $(12 \%)(p=0.001)$. In vitro mutation frequencies for $f b i A, f g d 1$ and $f b i B(19 \%, 7 \%$ and $2 \%$, respectively) were similar to our findings in mice.

Among the 99 unique mutations, all but one (an IS6110 insertion located in 85-bp upstream of the fbiC coding sequence in isolate KA-026a (Table 2 and Table S2) were found within the coding regions of the six genes. In total, 54\% (53/99) were non-synonymous point mutations (no synonymous point mutations were identified), 35\% (35/99) were insertions or deletions (indels), 
and $11 \%(11 / 99)$ were substitutions resulting in a stop codon. No significant difference in the distribution of point mutation and indels was found between ours and the in vitro study by Haver, et al, in which non-synonymous point mutations and indels were $50 \%(75 / 151)$ and $24 \%(36 / 151)$, respectively. However, the frequency of stop codon substitutions in the latter study $(26 \%, 40 / 151)$ was higher than that observed in the present study $(11 \%, 11 / 99)(p=0.004), 85 \%(34 / 40)$ of which were in $d d n$ in the latter study (22). Non-synonymous point mutations predominated relative to indels and stop codon mutations overall and in each gene except for ddn (Fig. 2 and Table S4). The frequency of point mutations was similar between BALB/c and C3HeB/FeJ mice $(56 \%$ versus $51 \%$ ) (Table 3). A higher frequency of indels occurred in C3HeB/FeJ compared to BALB/c mice ( $44 \%$ versus $28 \%$ ), while more stop codon mutations occurred in BALB/c, but these differences were not statistically significant. There was no clear association between dose of pretomanid and the type of mutation selected.

Comparing the 99 unique mutations identified in our study with the 151 unique mutations in 5 of the same genes selected in vitro (22), only 4 mutations were found in the same position. Only W79 stop (fbiA) and N336K (fbiC) mutations were found in both datasets while both T273 and $\mathrm{H} 190$ (fbiC) were mutated in the same position but with different mutations. As expected from the fact that most mutants could be isolated on plates containing $10 \mu \mathrm{g} / \mathrm{ml}$ of pretomanid, MICs determined against a small subset of isolates indicated high-level pretomanid resistance (Table S5 and Table 1 and 2). Taken together, these data illustrate the tremendous diversity of mutations capable of conferring high-level pretomanid resistance.

\section{Mutations in Rv2983 cause resistance to pretomanid and delamanid.}

To prove that mutations in Rv2983 are sufficient for nitroimidazole resistance, merodiploid complemented strains were constructed by introducing a copy of the wild type RV2983 gene into B101, an Rv2983 mutant (A198P), through site-specific integration (31, 32). Following 
confirmation of successful integration by Southern blot using a DIG-labeled RV2983 probe (Figs. S1A and S1B), susceptibility testing by $7 \mathrm{H} 9$ broth dilution confirmed significantly higher nitroimidazole MICs against the Rv2983 mutant (pretomanid and delamanid MICs of $32 \mu \mathrm{g} / \mathrm{ml}$ and $0.064-0.128 \mu \mathrm{g} / \mathrm{ml}$, respectively) and full restoration of susceptibility in the complemented strains (pretomanid and delamanid MICs of $0.25 \mu \mathrm{g} / \mathrm{ml}$ and $0.008 \mu \mathrm{g} / \mathrm{ml}$, respectively).

\section{$R v 2983$ is required for $F_{420}$ biosynthesis.}

To demonstrate that $R v 2983$ is required for $F_{420}$ biosynthesis, we measured the production of Fo and $\mathrm{F}_{420}$ in M. smegmatis strains overexpressing Rv2983 and in M. tuberculosis Rv2983 mutant strains compared with their corresponding control strains. Rv2983 and fbiC were successfully cloned into pYUBDuet and pfbiC (designated pRV2983 and pfbiC-Rv2983, respectively), followed by successful transformation of $M$. smegmatis, along with pYUBDuet and pfbiABC, which were confirmed by restriction enzyme digestion, DNA sequencing and PCR amplification (data not shown). Overexpression of Rv2983 in M. smegmatis increased $F_{420}$ production but resulted in little change in Fo production compared to the control strain after 6 and 26 hours of induction with IPTG (Figs. 3A and 3B). As expected, mutation of Rv2983 in the M. tuberculosis B101 mutant markedly reduced $\mathrm{F}_{420}$ production, resulting in accumulation of Fo. Complementation fully restored the wild-type phenotype (Figs. 3C and 3D). In order to evaluate the method, we also overexpressed $\mathrm{fbiC}$, which encodes the Fo synthase, with and without concomitant overexpression of Rv2983 in M. smegmatis. As expected, overexpression of fbiC increased Fo and, consequently, $F_{420}$ concentrations. Relative to the control strain, $F_{420}$ concentrations were similar when either fbiC or Rv2983 was over-expressed alone (Fig. 3A). Interestingly, when Rv2983 was co-overexpressed with fbic, a dramatic increase in $\mathrm{F}_{420}$ was observed relative to over-expression of either gene alone (3.4 and 3.1-fold, respectively) after 6 hours of IPTG induction $(p<0.001)$, with corresponding significant decreases of Fo levels after 6 and 26 hours of IPTG induction (5.8 and 3.1-fold; $p<0.005$ and 0.05 , respectively) (Fig. 3A and 3B). These results 
suggest that the excess Fo produced by fbiC over-expression was efficiently converted to $\mathrm{F}_{420}$ by over-expressed Rv2983. On the other hand, although a small amount of $F_{420}$ was observed in cell extracts of two Rv2983 point mutants (B101 [A198P] and KA016 [Q114R]), their $\mathrm{F}_{420}$ content was significantly lower than that of the wild type $(7.3$ and 7.7 -fold $)(p<0.001)$ and complemented B101 mutant (Fig. 3C). As expected, Fo accumulated in the two Rv2983 mutant strains relative the wildtype (6.7 and 6.5-fold; $p<0.05$ and 0.005 , respectively) (Fig. 3D), indicating that Fo was not efficiently converted to $\mathrm{F}_{420}$ in the presence of a mutated RV2983. Two other pretomanid-resistant strains were also assessed as controls. The KA026 mutant with an IS6110 insertion 85 bp upstream of fbiC had undetectable Fo and very little $\mathrm{F}_{420}$ content, while the KA91 mutant with an IS6110 insertion at amino acid position 108 of Ddn showed a wild-type phenotype with respect to $\mathrm{F}_{420}$ and Fo concentrations (Fig.3C and D).

To understand the effect of mutations on gene expression and its regulation, we performed RTqPCR after sub-culturing the M. tuberculosis strains in fresh 7H9 broth. Expression of Rv2983 and fbiC increased 2.4- and 1.6-fold, respectively, in the Rv2983 mutant B101 relative to the wildtype H37Rv parent after 4 days of incubation in 7H9 broth. Similar increases were observed in other genes such as fbiA (2.3-fold), fbiB (2.0-fold) and fgd1 (2.2-fold) involved in $\mathrm{F}_{420}$ biosynthesis, suggesting that the reduced $\mathrm{F}_{420}$ content caused by the Rv2983 A198P mutation resulted in upregulation of the $\mathrm{F}_{420}$ biosynthesis pathway (Fig. S2A). Expression of $f b i C$ in the KA026 mutant decreased 114-fold compared to that in the wild-type H37Rv parent after 2 days of incubation in 7H9 broth, likely resulting from interrupted fbiC transcription as a result of the insertion IS6110 at 85-bp upstream and explaining the low levels of both Fo and $F_{420}$ in that mutant (Fig. S2 B). A faint band representing the fbiC DNA fragment of 937-bp from the fbiC KA026 mutant relative to H37Rv further supports this conclusion (Fig. S2C).

\section{$F_{420}$-deficient pretomanid-resistant mutants are attenuated for growth in the presence of malachite green.}


Previous work using M. smegmatis showed that mutations in MSMEG_5126 (homolog of fbiC) and MSMEG_2392 (which shares 69\% homology with Rv2983) reduce the ability to decolorize and detoxify $M G$, indicating that $F_{420}$ is necessary for this process (20). To evaluate the role of each gene associated with nitroimidazole activation in the susceptibility to MG, log-phase cultures of 10 selected mutants were plated on $7 \mathrm{H} 9$ agar supplemented with a range of MG concentrations. All mutants deficient in $\mathrm{F}_{420}$ synthesis or reduction (i.e., those with mutations in fbiA-C, Rv2983 or $f g d 1$ ) were more susceptible to MG, while the ddn mutant retained the same susceptibility as the wild type H37Rv parent, whose growth was almost completely inhibited at MG concentrations $\geq 30 \mu \mathrm{g} / \mathrm{ml}$ (Fig. 4A). The lability of $\mathrm{F}_{420} \mathrm{H}_{2}$ and lack of a commercial source for $\mathrm{F}_{420}$ made it unfeasible to attempt to test whether provision of $\mathrm{F}_{420} \mathrm{H}_{2}$ could rescue the MGhypersusceptible phenotype of the $\mathrm{F}_{420} \mathrm{H}_{2}$-deficient mutants. To confirm that $R v 2983$ is necessary for the intrinsic resistance of M. tuberculosis to MG, we compared the growth of the B101 mutant to that of the wild type and complemented strains on MG. Again, growth of this Rv2983 mutant was inhibited by lower concentrations of MG than the wild type strain and required longer incubation times before colonies appeared on MG-containing plates (Fig. 4B-D). Plating at higher bacterial density $(500 \mu \mathrm{l}$ rather than $100 \mu \mathrm{l}$ of cell suspension per plate) significantly increased recovery (Fig. S3A and B). Complementation of Rv2983 fully restored the wild-type growth phenotype on MG concentrations up to $6 \mu \mathrm{g} / \mathrm{ml}$. Interestingly, at MG concentrations above 6 $\mu \mathrm{g} / \mathrm{ml}$, greater recovery was observed when Rv2983 was expressed behind the native promoter compared to the hsp60 promoter (Fig. 4B-D). Smaller colony size was observed in the Rv2983 mutant relative to the wild type and the complemented strains on plates without MG after 21 days of incubation but not after 28 days of incubation (Fig. 5). However, smaller colony size and deficient decolorization was observed for the Rv2983 mutant on plates containing MG concentrations as low as 0.25 and $1 \mu \mathrm{g} / \mathrm{ml}$, even after 28 days of incubation (Fig. 5). At MG concentrations of $6-12 \mu \mathrm{g} / \mathrm{ml}$, even more-concentrated aliquots of the B101 mutant culture showed markedly reduced recovery despite 42 days of incubation (Fig. 5). 
Because all solid media commonly used to isolate and cultivate M. tuberculosis in clinical laboratories contain MG as a selective decontaminant, the increased MG susceptibility conferred by mutations in fbiA-C, RV2983 and fgd1 could compromise the isolation and propagation (and hence identification) of nitroimidazole-resistant mutants from clinical samples. Commercial 7H10 agar, 7H11 agar and LJ medium contain $0.25,1$ and $400 \mu \mathrm{g} / \mathrm{ml}$, respectively, of MG. To assess the potential impact of these media on the isolation of an $\mathrm{F}_{420} \mathrm{H}_{2}$-deficient nitroimidazole-resistant Rv2983 mutant relative to an $\mathrm{F}_{420} \mathrm{H}_{2}$-sufficient, but still nitroimidazole-resistant, ddn mutant and the nitroimidazole-susceptible wild type and Rv2983-complemented mutant, we inoculated these media in parallel using serial dilutions of each strain. The Rv2983 mutant exhibited 10 times lower CFU counts and smaller colony size relative to other strains after 21 and 28 days of incubation on $7 \mathrm{H} 10$ agar plates $(p<0.01)$ (Figs. $6 \mathrm{~A}$ and $\mathrm{C}(\mathrm{a}))$. The result after 35 days of incubation was generally similar between the mutant and the control strains (Fig. 6A and $C(b)$ ). A similar semiquantitative growth assessment of the RV2983 mutant on LJ media compared to other strains including a ddn mutant (K91, IS6110 ins in D108) revealed growth inhibition of the RV2983 mutant that was ameliorated by increasing the size of the bacterial inoculum from $10^{2}$ to $10^{6} \mathrm{CFU} / \mathrm{ml}$ and increasing the incubation time from 28 to 35 days (Fig. 6D). Interestingly, no difference in growth was found on $7 \mathrm{H} 11$ agar (Fig. 6B), even when comparing colony size after just 21 days of incubation (Fig. 6C(c)), despite higher MG concentrations in that medium compared to $7 \mathrm{H} 10$. Unlike 7H10, 7H11 medium contains hydrolysate of casein, which may somehow mitigate against the MG toxicity. Taken together, these results indicate that use of $7 \mathrm{H} 10$ and $\mathrm{LJ}$ could compromise the recovery and propagation of $\mathrm{F}_{420} \mathrm{H}_{2}$-deficient nitroimidazole-resistant mutants from clinical specimens and that $7 \mathrm{H} 11$ agar may be the preferred solid media for processing specimens propagating any isolates from nitroimidazole-treated patients. 


\section{DISCUSSION}

As representatives of one of only two new drug classes approved for use against TB in roughly 50 years, delamanid and pretomanid are important and promising new drugs $(3,4,6,7,39)$. The former received accelerated approval from the EMA for treatment of MDR-TB and is now used clinically, albeit sparingly, while a phase 3 trial is being completed. The latter is being evaluated in clinical trials as a component of highly promising regimens for both drug-susceptible and MDR/XDR-TB. Comprehensive knowledge of genetic mutations conferring nitroimidazole resistance in $M$. tuberculosis and the resultant mutant phenotypes are critical for timely and accurate diagnosis of resistance and, therefore, the safe and effective use of these drugs in the clinical setting. Previous work identified 5 genes ( $f b i A-C$, fgd1, and $d d n$ ) involved in the activation pathway of nitroimidazole prodrugs in which mutations may confer drug resistance in $M$. tuberculosis complex $(16,18,21-23,40)$. In a prior study of the spectrum of nitroimidazole resistance-conferring mutations, Haver, et al found that 151 (83\%) of 183 pretomanidresistant isolates selected in vitro harbored a single mutation in one of these 5 genes (22). However, $17 \%$ of the selected strains harbored no mutations in these genes. The present study has several important new findings. First, we identified a novel nitroimidazole resistance determinant—mutations in Rv2983—-that explained, in the case of our study, all of the pretomanid resistance that was not attributable to mutations in the 5 previously described genes. Second, we demonstrate for the first time that $R v 2983$ is required for $\mathrm{F}_{420}$ biosynthesis and likely plays a role similar to cofC in the methanogen M. jannaschii (19). Third, we show that Rv2983 and the ability to produce $\mathrm{F}_{420} \mathrm{H}_{2}$ are essential for full tolerance of $M$. tuberculosis to the selective decontaminant MG, which raises serious concerns about the ability to reliably recover most nitroimidazoleresistant mutants from clinical samples on the most widely used microbiology media.

Our study provides the first comprehensive analysis of the spectrum of nitroimidazole-resistant mutants selected in vivo and, because we used WGS, it represents the most comprehensive 
analysis made to-date. Despite the insignificant differences in mutation frequencies between the two mouse strains for other genes related to nitroimidazole activation, Rv2983 mutations were observed with a somewhat higher frequency in BALB/c mice, indicating a possible host-specific fitness cost that warrants further dedicated study. Like the in vitro study by Haver et al (22), we found that isolated mutations in fbiA-C, fgd1, or $d d n$ explained the majority of the pretomanidresistant isolates we selected. However, whereas their study left $17 \%$ of resistant isolates unexplained, we found that all of the remaining resistant isolates, representing $10 \%$ of the total number of unique mutations, harbored mutations in Rv2983, a gene not previously implicated in nitroimidazole resistance. Indeed, the proportion of resistant isolates explained by RV2983 (10\%) was similar to the proportion explained by fbiA (15\%) and $d d n(12 \%)$ mutations, which lagged only mutations in fbic (55\%) as the predominant cause of pretomanid resistance in our mice. Although the Rv2983 A198T mutation caused a smaller upward shift in the delamanid MIC compared to the pretomanid MIC, the delamanid MIC of $0.064-0.128 \mu \mathrm{g} / \mathrm{ml}$ against the mutant was still significantly higher than the recently proposed critical concentration of $0.016 \mu \mathrm{g} / \mathrm{ml}(41)$. Thus, the identification of Rv2983 mutations should be included in rapid molecular drug susceptibility tests and algorithms for the diagnosis of nitroimidazole resistance from genome sequence data. The 10 mutations in Rv2983 identified in this study (Table 1 and 2) represent the first step in the process of identifying specific resistance-conferring mutations to inform test development.

Rv2983 has $22 \%$ amino acid sequence identity to CofC (MJ0887) of M. jannaschii, a putative guanylyltransferase involved in the biosynthesis of coenzyme $\mathrm{F}_{420}$ from the precursor $\mathrm{Fo}$. CofC is believed to catalyze the condensation of 2-phospho-L-lactate (LP) and GTP to form lactyl-2diphospho-5'-guanosine (LPPG) and PP $\mathrm{i}_{\mathrm{i}}$ (19). Subsequent condensation of LPPG with Fo catalyzed by $\operatorname{CofD}(\mathrm{FbiA})$ forms $\mathrm{F}_{420}-0$ and is followed by sequential addition of glutamate residues by CofE $(\mathrm{FbiB})$ to produce $\mathrm{F}_{420}$ with a poly-glutamate tail (19). Up to now, a CofC homolog 
has not been identified in M. tuberculosis. It is known that Fo biosynthesis is catalyzed by FbiC, but it is not clear how Fo is modified by FbiA to form $\mathrm{F}_{420}-0$. Bashiri, et al tried to understand the mechanism by overexpressing fbiC in the saprophyte $M$. smegmatis, which did not dramatically increase $F_{420}$ biosynthesis, suggesting that an additional intermediate is required to form $F_{420}-0$ from Fo (35). Using overexpression of Rv2983 in M. smegmatis and M. tuberculosis Rv2983 mutants, we provide initial evidence that Rv2983 catalyzes an important step required for synthesis of $F_{420}$ from Fo in the pathogen M. tuberculosis, which adds to previous evidence that its ortholog MSMEG_2392 is involved in $\mathrm{F}_{420}$ biosynthesis in M. smegmatis (20). The validity of the method used in this study for detection of $F_{420}$ and Fo was demonstrated by showing the expected results with two pretomanid-resistant strains, KA016 and KA026, harboring mutations in fbiC and $d d n$, respectively. Although the role of Rv2983 remains to be confirmed, our results confirm that expression of Rv2983 is necessary for efficient conversion of Fo to $F_{420}$ (Fig. 3G).

Our results confirm and significantly extend prior in vitro work demonstrating the remarkable diversity of mutations capable of conferring high-level nitroimidazole resistance. Among the 99 unique mutations we identified in 47 mice, only 3 mutations (K9N in fgd1, R322L in fbiC and Q120P in fbiA) were found in more than one mouse. Furthermore, by comparing the 99 unique mutations observed in our mice with the 151 unique mutations selected in vitro (22), the same mutation occurred only twice. Thus, each of the 6 genes now implicated in nitroimidazole resistance appears to be devoid of "hot spots" for such mutations. The frequency of spontaneous mutations conferring nitroimidazole resistance in $M$. tuberculosis has been investigated in vitro by several studies and found to range from 1 in $10^{5}$ to 7 in $10^{7} \mathrm{CFU}(21-23,27,40,42)$, which is consistent with our findings in the lungs of untreated BALB/C mice. The large "target size" for mutations in 6 non-essential genes drives these high frequencies of spontaneous nitroimidazoleresistant mutants in $M$. tuberculosis populations, which is as high or higher than that for isoniazid and other TB drugs in clinical use. Our unpublished observations suggest that similar frequencies 
of nitroimidazole-resistant mutants exist in sputum isolates collected from treatment-naïve, drugsusceptible TB patients. Delamanid-resistant $M$. tuberculosis has been recovered from patients both before and after delamanid treatment $(10,43-45)$. To date, emergence of resistance has not been described during use of pretomanid in clinical trials, but such use has been restricted to relatively short treatment durations and/or use of highly active companion drugs. Pretomanid resistance has emerged during combination therapy in mouse models $(3,46)$. Thus, the relatively high frequency of spontaneous mutations conferring nitroimidazole resistance and available preclinical and clinical data underscore the importance of making validated drug susceptibility testing for this class widely available as clinical usage expands. The unprecedented number and diversity of resistance-conferring mutations demonstrated for nitroimidazole drugs here and by Haver et al (22), clearly challenges the development and interpretation of rapid molecular susceptibility tests, especially considering that polymorphisms in nitroimidazole resistance genes that represent phylogenetic markers but do not confer pretomanid resistance are well-described $(47,48)$. A similar situation exists for pncA mutations and pyrazinamide (PZA) resistance, where an efficient, yet comprehensive method based on saturating mutagenesis for distinguishing single nucleotide polymorphisms conferring resistance was recently described (49). A similar analysis of substitutions in the 6 genes related to nitroimidazole resistance would similarly advance the development of drug susceptibility testing using genome sequencing technology.

Amino acid substitutions in the PZA-activating PncA protein that confer PZA resistance are associated with diminished enzymatic activity or reduced abundance of the protein (49). Similar mechanisms could explain the overall mechanisms of the 99 mutations we identified in the six genes conferring nitroimidazole resistance. The Rv2983 A198P or Q114R substitutions may change the conformation of Rv2983 protein structure and reduce its enzymatic activity given that it has no effect on Rv2983 gene transcription. $F_{420}$ biosynthesis is dramatically decreased while expression of Rv2983 and other genes increases, perhaps indicating an unknown regulatory 
mechanism triggered by reduced $\mathrm{F}_{420}$ biosynthesis. Mutations identified in Rv2983 in this study will provide useful information for future studies on structure-function associations of the potential M. tuberculosis CofC homolog. The KA026 mutant harboring an IS6110 insertion 85 bp upstream of fbiC exhibited significantly decreased expression of fbic, possibly due to interruption of the promoter region, and, consequently, almost complete loss of Fo synthase activity. Hence, the upstream regulatory regions of the six genes also should be considered in future identification of resistance-conferring mutations, although their prevalence in this study was only $1 \%$.

Our findings regarding the heightened susceptibility of $\mathrm{F}_{420} \mathrm{H}_{2}$-deficient mutants to $\mathrm{MG}$ pose a previously unappreciated challenge to the development and use of phenotypic testing methods. Indeed, we observed reduced or delayed recovery of a nitroimidazole-resistant Rv2983 mutant on commercial 7H10 and LJ media that include MG as a selective decontaminant (50). Since fbiA$C$ and fgd1 mutants, as well as a second Rv2983 mutant, exhibited similar hypersusceptibility to MG in $7 \mathrm{H} 9$ agar supplemented with MG, their recovery on $7 \mathrm{H} 10$ and $\mathrm{LJ}$ is also likely to be affected. Selective growth inhibition of nitroimidazole-resistant strains on media that are commonly used in clinical microbiology laboratories around the world raises serious concern that their recovery from clinical specimens may be impaired to such an extent that it reduces the sensitivity and accuracy of phenotypic drug susceptibility testing, especially for isolates comprised of mixed wild-type and resistant populations. This concern is further amplified by the common practice of performing susceptibility testing (including molecular testing), not on primary samples but, on isolates that have been sub-cultured one or more times on solid media. Such practices may drastically reduce the proportion of (or eradicate) $\mathrm{F}_{420} \mathrm{H}_{2}$-deficient mutants present in the original sample. In addition, efforts to develop MG decolorization assays for detection of drug-resistant TB are expected to be fruitless for these mutants (51-54). We did not determine the basis for the greater recovery of $\mathrm{F}_{420} \mathrm{H}_{2}$-deficient mutants on $7 \mathrm{H} 11$ vs. $7 \mathrm{H} 10$ media despite $4 x$ higher total MG concentrations in the former. The principal differences between these media are the presence of pancreatic digest 
of casein in $7 \mathrm{H} 11$ and lower concentrations of magnesium sulfate countered by the addition of copper sulfate, zinc sulfate and calcium chloride in $7 \mathrm{H} 10$. Although this issue clearly requires further study, we presently believe that $7 \mathrm{H} 10$ and LJ should not be employed for phenotypic nitroimidazole susceptibility testing and that primary isolation or subculture of any isolate on such media prior to either phenotypic or genotypic susceptibility testing should be avoided whenever possible. When it cannot be avoided, larger inoculum sizes and longer incubation times may increase recovery on $7 \mathrm{H} 10$ and LJ. Based on our study, $7 \mathrm{H} 11$ agar appears to be the preferred solid medium for recovery of $\mathrm{F}_{420} \mathrm{H}_{2}$-deficient nitroimidazole-resistant $M$. tuberculosis.

A role for cofactor $\mathrm{F}_{420} \mathrm{H}_{2}$ in tolerance of was previously demonstrated (20). Whereas mycobacteria are normally capable of decolorizing and detoxifying MG, mutations in the saprophyte M. smegmatis orthologs of fgd1, fbiC and Rv2983 disrupt this ability and/or reduce the MIC of MG $(20,55)$. We now extend these observations to the pathogen M. tuberculosis and also implicate $f b i A$ and $f b i B$, but not $d d n$, in MG tolerance. In Citrobacter species, an NADHdependent triphenylmethane reductase catalyzes reduction of MG to colorless leucoMG that lacks antimicrobial activity and is sequestered in the lipid fraction of the cells $(56,57)$. Triphenylmethane reductase has not been identified in mycobacteria. However, the enhanced MG susceptibility of the $\mathrm{F}_{420} \mathrm{H}_{2}$-deficient mutants, but not ddn mutants, suggests that one or more analogous, yet unidentified, $\mathrm{F}_{420} \mathrm{H}_{2}$-dependent reductases is responsible for decolorizing and detoxifying MG in mycobacteria.

Prior studies indicated other fitness costs associated with $\mathrm{F}_{420} \mathrm{H}_{2}$ deficiency, such as hypersusceptibility to oxidative and nitrosative stresses $(12,58,59)$. Nevertheless, we observed selective amplification of $\mathrm{F}_{420} \mathrm{H}_{2}$-deficient mutants in mice over a range of pretomanid doses that included doses producing much higher drug exposures than those produced in patients. Amplification was especially pronounced at higher drug doses, which eliminated the majority of nitroimidazole-susceptible population more effectively, and in C3HeB/FeJ mice. The lack of 
marked in vivo fitness defects is also supported by the fact that the proportion of all unique resistance mutations explained by each mutation did not differ much between the in vitro and in vivo conditions, except that a higher proportion of $f b i C$ mutations predominated in mice, somewhat at the expense of $d d n$ mutations (22). Likewise, indels and stop codon mutations comprised nearly $50 \%$ of the mutations responsible for resistance. This proportion may be biased due to the high drug doses tested in some mice because such mutations are more likely to result in complete loss-of-function and therefore more complete resistance. However, the frequency of such mutations did not appear to change in a dose-dependent manner. Interestingly, most Rv2983 mutants were selected in BALB/c rather than $\mathrm{C} 3 \mathrm{HeB} / \mathrm{FeJ}$ mice despite similar mutation rates in other genes between the two mouse strains. Whether this represents a real or a random difference will require further study. Clearly, clinicians must rely on effective combination drug therapy to prevent the selective amplification of nitroimidazole-resistant mutants. Heightened susceptibility to agents causing oxidative stress has been demonstrated for $\mathrm{F}_{420} \mathrm{H}_{2}$-deficient $M$. tuberculosis and $M$. smegmatis mutants (58). A more complete understanding of the unique vulnerabilities of such mutants should aid in the design of effective combination regimens that also optimally restrict selection of nitroimidazole-resistant mutants.

In conclusion, using BALB/C and $\mathrm{C} 3 \mathrm{HeB} / \mathrm{FeJ}$ mice and WGS, we characterized the pretomanid dose-response relationships for bactericidal effect and suppression of drug-resistant mutants and profiled the genetic spectrum of pretomanid resistance emerging in vivo. A novel resistance determinant, Rv2983, was identified as essential for $F_{420}$ biosynthesis and activation of the novel TB pro-drugs delamanid and pretomanid. Furthermore, we provide evidence that $\mathrm{F}_{420} \mathrm{H}_{2}$-deficient, nitroimidazole-resistant $M$. tuberculosis mutants are hypersensitive to MG, raising concern that using MG-containing medium could compromise the isolation and propagation of M. tuberculosis from clinical samples and therefore hinder the clinical diagnosis of nitroimidazole resistance. These findings have important implications for both genotypic and phenotypic susceptibility 
testing to detect nitroimidazole resistance, which will be of increasing importance as wider use of delamanid and, if approved, pretomanid, ensues.

\section{LIST OF SUPPLEMENTARY MATERIALS}

Table S1

Table S2

Table S3

Table S4

Table S5

Figure S1

Figure S2

Figure S3

Acknowledgements: The Global Alliance for TB Drug Development kindly provided pretomanid and delamanid. Funding: The authors gratefully acknowledge support in the form of funding from the Bill and Melinda Gates Foundation (OPP1037174) (ELN) and the National Institutes of Health (R01-Al111992) (ELN). G.B. is supported by a Sir Charles Hercus Fellowship through the Health Research Council of New Zealand. Author contributions: D.R. and E.N. conceived the study and designed the experiments. S.L. and J.L. assisted with the design and conduct of the in vivo experiment. Whole genome sequencing was performed and analyzed by T.I., J.S., D.R. and E.N. In vitro experiments were performed and analyzed by D.R., J.L. and E.N. The manuscript was drafted by D.R. and E.N. with critical input from T.I., J.S., and G.B. Competing interests: All authors declare that they have no competing interests. Data and materials availability: All data necessary for evaluation of the conclusions are present in the paper and/or the Supplementary Materials. Pretomanid and delamanid were provided under a material transfer agreement. 


\section{REFERENCES}

1. WHO, Global Tuberculosis report. World Health Organization Geneva, (2017).

2. WHO, Guidelines for the programmatic management of drug-resistant tuberculosis. . World Health Organization Geneva, (2011).

3. S. Y. Li, R. Tasneen, S. Tyagi, H. Soni, P. J. Converse, K. Mdluli, E. L. Nuermberger, Bactericidal and Sterilizing Activity of a Novel Regimen with Bedaquiline, Pretomanid, Moxifloxacin, and Pyrazinamide in a Murine Model of Tuberculosis. Antimicrobial agents and chemotherapy 61, (2017).

4. R. Tasneen, F. Betoudji, S. Tyagi, S. Y. Li, K. Williams, P. J. Converse, V. Dartois, T. Yang, C. M. Mendel, K. E. Mdluli, E. L. Nuermberger, Contribution of Oxazolidinones to the Efficacy of Novel Regimens Containing Bedaquiline and Pretomanid in a Mouse Model of Tuberculosis. Antimicrobial agents and chemotherapy 60, 270-277 (2015).

5. V. Skripconoka, M. Danilovits, L. Pehme, T. Tomson, G. Skenders, T. Kummik, A. Cirule, V. Leimane, A. Kurve, K. Levina, L. J. Geiter, D. Manissero, C. D. Wells, Delamanid improves outcomes and reduces mortality in multidrug-resistant tuberculosis. Eur Respir J 41, 1393-1400 (2013).

6. M. T. Gler, V. Skripconoka, E. Sanchez-Garavito, H. Xiao, J. L. Cabrera-Rivero, D. E. Vargas-Vasquez, M. Gao, M. Awad, S. K. Park, T. S. Shim, G. Y. Suh, M. Danilovits, H. Ogata, A. Kurve, J. Chang, K. Suzuki, T. Tupasi, W. J. Koh, B. Seaworth, L. J. Geiter, C. D. Wells, Delamanid for multidrug-resistant pulmonary tuberculosis. N Engl J Med 366, 2151-2160 (2012).

7. A. H. Diacon, R. Dawson, F. von Groote-Bidlingmaier, G. Symons, A. Venter, P. R. Donald, C. van Niekerk, D. Everitt, H. Winter, P. Becker, C. M. Mendel, M. K. Spigelman, 14-day bactericidal activity of PA-824, bedaquiline, pyrazinamide, and moxifloxacin combinations: a randomised trial. Lancet 380, 986-993 (2012).

8. R. Dawson, A. H. Diacon, D. Everitt, C. van Niekerk, P. R. Donald, D. A. Burger, R. Schall, M. Spigelman, A. Conradie, K. Eisenach, A. Venter, P. Ive, L. Page-Shipp, E. Variava, K. Reither, N. E. Ntinginya, A. Pym, F. von Groote-Bidlingmaier, C. M. Mendel, Efficiency and safety of the combination of moxifloxacin, pretomanid (PA-824), and pyrazinamide during the first 8 weeks of antituberculosis treatment: a phase $2 \mathrm{~b}$, openlabel, partly randomised trial in patients with drug-susceptible or drug-resistant pulmonary tuberculosis. Lancet 385, 1738-1747 (2015).

9. M. Matsumoto, H. Hashizume, T. Tomishige, M. Kawasaki, H. Tsubouchi, H. Sasaki, Y. Shimokawa, M. Komatsu, OPC-67683, a nitro-dihydro-imidazooxazole derivative with promising action against tuberculosis in vitro and in mice. PLoS medicine 3, e466 (2006).

10. European Medicines Agency, Summary of the European public assessment report (EPAR) for Deltyba. Found at http://www.ema.europa.eu/docs/en_GB/document_library/EPAR__Public_assessment_report/human/002552/WC500166234.pdf (2013). Last accessed on 18 June 2018.

11. U. Manjunatha, H. I. Boshoff, C. E. Barry, The mechanism of action of PA-824: Novel insights from transcriptional profiling. Commun Integr Biol 2, 215-218 (2009).

12. C. Greening, F. H. Ahmed, A. E. Mohamed, B. M. Lee, G. Pandey, A. C. Warden, C. Scott, J. G. Oakeshott, M. C. Taylor, C. J. Jackson, Physiology, Biochemistry, and Applications of F420- and Fo-Dependent Redox Reactions. Microbiol Mol Biol Rev 80, 451-493 (2016).

13. S. E. Cellitti, J. Shaffer, D. H. Jones, T. Mukherjee, M. Gurumurthy, B. Bursulaya, H. I. Boshoff, I. Choi, A. Nayyar, Y. S. Lee, J. Cherian, P. Niyomrattanakit, T. Dick, U. H. Manjunatha, C. E. Barry, 3rd, G. Spraggon, B. H. Geierstanger, Structure of Ddn, the 
deazaflavin-dependent nitroreductase from Mycobacterium tuberculosis involved in bioreductive activation of PA-824. Structure 20, 101-112 (2012).

14. G. Bashiri, C. J. Squire, N. J. Moreland, E. N. Baker, Crystal structures of F420dependent glucose-6-phosphate dehydrogenase FGD1 involved in the activation of the anti-tuberculosis drug candidate PA-824 reveal the basis of coenzyme and substrate binding. The Journal of biological chemistry 283, 17531-17541 (2008).

15. D. E. Graham, H. Xu, R. H. White, Identification of the 7,8-didemethyl-8-hydroxy-5deazariboflavin synthase required for coenzyme F(420) biosynthesis. Arch Microbiol 180, 455-464 (2003).

16. K. P. Choi, T. B. Bair, Y. M. Bae, L. Daniels, Use of transposon Tn5367 mutagenesis and a nitroimidazopyran-based selection system to demonstrate a requirement for fbiA and fbiB in coenzyme $F(420)$ biosynthesis by Mycobacterium bovis BCG. J Bacteriol 183, 7058-7066 (2001).

17. L. Decamps, B. Philmus, A. Benjdia, R. White, T. P. Begley, O. Berteau, Biosynthesis of F0, precursor of the F420 cofactor, requires a unique two radical-SAM domain enzyme and tyrosine as substrate. J Am Chem Soc 134, 18173-18176 (2012).

18. K. P. Choi, N. Kendrick, L. Daniels, Demonstration that $\mathrm{fbiC}$ is required by Mycobacterium bovis BCG for coenzyme F(420) and FO biosynthesis. J Bacteriol 184, 2420-2428 (2002).

19. L. L. Grochowski, H. Xu, R. H. White, Identification and characterization of the 2phospho-L-lactate guanylyltransferase involved in coenzyme F420 biosynthesis. Biochemistry 47, 3033-3037 (2008).

20. D. Guerra-Lopez, L. Daniels, M. Rawat, Mycobacterium smegmatis mc2 $155 \mathrm{fbiC}$ and MSMEG_2392 are involved in triphenylmethane dye decolorization and coenzyme F420 biosynthesis. Microbiology 153, 2724-2732 (2007).

21. C. K. Stover, P. Warrener, D. R. VanDevanter, D. R. Sherman, T. M. Arain, M. H. Langhorne, S. W. Anderson, J. A. Towell, Y. Yuan, D. N. McMurray, B. N. Kreiswirth, C. E. Barry, W. R. Baker, A small-molecule nitroimidazopyran drug candidate for the treatment of tuberculosis. Nature 405, 962-966 (2000).

22. H. L. Haver, A. Chua, P. Ghode, S. B. Lakshminarayana, A. Singhal, B. Mathema, R. Wintjens, P. Bifani, Mutations in genes for the F420 biosynthetic pathway and a nitroreductase enzyme are the primary resistance determinants in spontaneous in vitroselected PA-824-resistant mutants of Mycobacterium tuberculosis. Antimicrobial agents and chemotherapy 59, 5316-5323 (2015).

23. M. Fujiwara, M. Kawasaki, N. Hariguchi, Y. Liu, M. Matsumoto, Mechanisms of resistance to delamanid, a drug for Mycobacterium tuberculosis. Tuberculosis (Edinb) 108, 186-194 (2018).

24. S. Subbian, L. Tsenova, M. J. Kim, H. C. Wainwright, A. Visser, N. Bandyopadhyay, J. S. Bader, P. C. Karakousis, G. B. Murrmann, L. G. Bekker, D. G. Russell, G. Kaplan, Lesion-Specific Immune Response in Granulomas of Patients with Pulmonary Tuberculosis: A Pilot Study. PLoS One 10, e0132249 (2015).

25. D. Rifat, B. Prideaux, R. M. Savic, M. E. Urbanowski, T. L. Parsons, B. Luna, M. A. Marzinke, A. A. Ordonez, V. P. DeMarco, S. K. Jain, V. Dartois, W. R. Bishai, K. E. Dooley, Pharmacokinetics of rifapentine and rifampin in a rabbit model of tuberculosis and correlation with clinical trial data. Science translational medicine 10, (2018).

26. E. L. Nuermberger, T. Yoshimatsu, S. Tyagi, K. Williams, I. Rosenthal, R. J. O'Brien, A. A. Vernon, R. E. Chaisson, W. R. Bishai, J. H. Grosset, Moxifloxacin-containing regimens of reduced duration produce a stable cure in murine tuberculosis. American journal of respiratory and critical care medicine 170, 1131-1134 (2004). 
27. S. Tyagi, E. Nuermberger, T. Yoshimatsu, K. Williams, I. Rosenthal, N. Lounis, W. Bishai, J. Grosset, Bactericidal activity of the nitroimidazopyran PA-824 in a murine model of tuberculosis. Antimicrobial agents and chemotherapy 49, 2289-2293 (2005).

28. M. H. Larsen, K. Biermann, S. Tandberg, T. Hsu, W. R. Jacobs, Jr., Genetic Manipulation of Mycobacterium tuberculosis. Current protocols in microbiology Chapter 10, Unit 10A 12 (2007).

29. T. R. loerger, Y. Feng, K. Ganesula, X. Chen, K. M. Dobos, S. Fortune, W. R. Jacobs, Jr., V. Mizrahi, T. Parish, E. Rubin, C. Sassetti, J. C. Sacchettini, Variation among genome sequences of H37Rv strains of Mycobacterium tuberculosis from multiple laboratories. J Bacteriol 192, 3645-3653 (2010).

30. T. R. loerger, T. O'Malley, R. Liao, K. M. Guinn, M. J. Hickey, N. Mohaideen, K. C. Murphy, H. I. Boshoff, V. Mizrahi, E. J. Rubin, C. M. Sassetti, C. E. Barry, 3rd, D. R. Sherman, T. Parish, J. C. Sacchettini, Identification of new drug targets and resistance mechanisms in Mycobacterium tuberculosis. PLoS One 8, e75245 (2013).

31. M. H. Lee, L. Pascopella, W. R. Jacobs, Jr., G. F. Hatfull, Site-specific integration of mycobacteriophage L5: integration-proficient vectors for Mycobacterium smegmatis, Mycobacterium tuberculosis, and bacille Calmette-Guerin. Proceedings of the National Academy of Sciences of the United States of America 88, 3111-3115 (1991).

32. D. Rifat, D. A. Belchis, P. C. Karakousis, senX3-independent contribution of regX3 to Mycobacterium tuberculosis virulence. BMC microbiology 14, 265 (2014).

33. D. Almeida, T. loerger, S. Tyagi, S. Y. Li, K. Mdluli, K. Andries, J. Grosset, J. Sacchettini, E. Nuermberger, Mutations in pepQ Confer Low-Level Resistance to Bedaquiline and Clofazimine in Mycobacterium tuberculosis. Antimicrobial agents and chemotherapy 60, 4590-4599 (2016).

34. Z. Ahmad, C. A. Peloquin, R. P. Singh, H. Derendorf, S. Tyagi, A. Ginsberg, J. H. Grosset, E. L. Nuermberger, PA-824 exhibits time-dependent activity in a murine model of tuberculosis. Antimicrobial agents and chemotherapy 55, 239-245 (2011).

35. G. Bashiri, A. M. Rehan, D. R. Greenwood, J. M. Dickson, E. N. Baker, Metabolic engineering of cofactor F420 production in Mycobacterium smegmatis. PLoS One 5, 0015803 (2010).

36. D. Isabelle, D. R. Simpson, L. Daniels, Large-Scale Production of Coenzyme F(420)-5,6 by Using Mycobacterium smegmatis. Applied and environmental microbiology 68, 57505755 (2002).

37. T. B. Bair, D. W. Isabelle, L. Daniels, Structures of coenzyme F(420) in Mycobacterium species. Arch Microbiol 176, 37-43 (2001).

38. D. Rifat, P. C. Karakousis, Differential regulation of the two-component regulatory system senX3-regX3 in Mycobacterium tuberculosis. Microbiology 160, 1125-1133 (2014).

39. R. Dawson, A. H. Diacon, D. Everitt, C. van Niekerk, P. R. Donald, D. A. Burger, R. Schall, M. Spigelman, A. Conradie, K. Eisenach, A. Venter, P. Ive, L. Page-Shipp, E. Variava, K. Reither, N. E. Ntinginya, A. Pym, F. von Groote-Bidlingmaier, C. M. Mendel, Efficiency and safety of the combination of moxifloxacin, pretomanid (PA-824), and pyrazinamide during the first 8 weeks of antituberculosis treatment: a phase $2 \mathrm{~b}$, openlabel, partly randomised trial in patients with drug-susceptible or drug-resistant pulmonary tuberculosis. Lancet $\mathbf{3 8 5}, 1738-1747$ (2015).

40. U. H. Manjunatha, H. Boshoff, C. S. Dowd, L. Zhang, T. J. Albert, J. E. Norton, L. Daniels, T. Dick, S. S. Pang, C. E. Barry, 3rd, Identification of a nitroimidazo-oxazinespecific protein involved in PA-824 resistance in Mycobacterium tuberculosis. Proceedings of the National Academy of Sciences of the United States of America 103, 431-436 (2006). 
41. WHO, Technical report on critical concentrations for TB drug susceptibility testing of medicines used in the treatment of drug-resistant TB.

http://www.who.int/tb/publications/2018/WHO_technical_report_concentrations_TB_drug _susceptibility, (2018).

42. J. G. Hurdle, R. B. Lee, N. R. Budha, E. I. Carson, J. Qi, M. S. Scherman, S. H. Cho, M. R. McNeil, A. J. Lenaerts, S. G. Franzblau, B. Meibohm, R. E. Lee, A microbiological assessment of novel nitrofuranylamides as anti-tuberculosis agents. J Antimicrob Chemother 62, 1037-1045 (2008).

43. G. V. Bloemberg, P. M. Keller, D. Stucki, A. Trauner, S. Borrell, T. Latshang, M. Coscolla, T. Rothe, R. Homke, C. Ritter, J. Feldmann, B. Schulthess, S. Gagneux, E. C. Bottger, Acquired Resistance to Bedaquiline and Delamanid in Therapy for Tuberculosis. (N Engl J Med. 2015 Nov 12;373(20):1986-8. doi: 10.1056/NEJMc1505196.).

44. K. Stinson, N. Kurepina, A. Venter, M. Fujiwara, M. Kawasaki, J. Timm, E. Shashkina, B. N. Kreiswirth, Y. Liu, M. Matsumoto, L. Geiter, MIC of Delamanid (OPC-67683) against Mycobacterium tuberculosis Clinical Isolates and a Proposed Critical Concentration. Antimicrobial agents and chemotherapy 60, 3316-3322 (2016).

45. H. Hoffmann, T. A. Kohl, S. Hofmann-Thiel, M. Merker, P. Beckert, K. Jaton, L. Nedialkova, E. Sahalchyk, T. Rothe, P. M. Keller, S. Niemann, Delamanid and Bedaquiline Resistance in Mycobacterium tuberculosis Ancestral Beijing Genotype Causing Extensively Drug-Resistant Tuberculosis in a Tibetan Refugee. American journal of respiratory and critical care medicine 193, 337-340 (2016).

46. J. Harper, C. Skerry, S. L. Davis, R. Tasneen, M. Weir, I. Kramnik, W. R. Bishai, M. G. Pomper, E. L. Nuermberger, S. K. Jain, Mouse model of necrotic tuberculosis granulomas develops hypoxic lesions. The Journal of infectious diseases $\mathbf{2 0 5}, \mathbf{5 9 5 - 6 0 2}$ (2012).

47. E. Schena, L. Nedialkova, E. Borroni, S. Battaglia, A. M. Cabibbe, S. Niemann, C. Utpatel, M. Merker, A. Trovato, S. Hofmann-Thiel, H. Hoffmann, D. M. Cirillo, Delamanid susceptibility testing of Mycobacterium tuberculosis using the resazurin microtitre assay and the BACTEC MGIT 960 system. J Antimicrob Chemother 71, 1532-1539 (2016).

48. S. Feuerriegel, C. U. Koser, D. Bau, S. Rusch-Gerdes, D. K. Summers, J. A. Archer, M. A. Marti-Renom, S. Niemann, Impact of Fgd1 and ddn diversity in Mycobacterium tuberculosis complex on in vitro susceptibility to PA-824. Antimicrobial agents and chemotherapy 55, 5718-5722 (2011).

49. A. N. Yadon, K. Maharaj, J. H. Adamson, Y. P. Lai, J. C. Sacchettini, T. R. loerger, E. J. Rubin, A. S. Pym, A comprehensive characterization of PncA polymorphisms that confer resistance to pyrazinamide. Nature communications 8, 588 (2017).

50. D. V. Cousins, B. R. Francis, B. L. Gow, Advantages of a new agar medium in the primary isolation of Mycobacterium bovis. Veterinary microbiology 20, 89-95 (1989).

51. N. C. Mirabal, S. L. Yzquierdo, D. Lemus, M. Madruga, Y. Milian, M. Echemendia, H. Takiff, A. Martin, P. Van der Stuyf, J. C. Palomino, E. Montoro, Evaluation of colorimetric methods using nicotinamide for rapid detection of pyrazinamide resistance in Mycobacterium tuberculosis. J Clin Microbiol 48, 2729-2733 (2010).

52. A. Y. Coban, M. Uzun, Rapid detection of multidrug-resistant Mycobacterium tuberculosis using the malachite green decolourisation assay. Mem Inst Oswaldo Cruz 108, 1021-1023 (2013).

53. A. Martin, F. Portaels, J. C. Palomino, Colorimetric redox-indicator methods for the rapid detection of multidrug resistance in Mycobacterium tuberculosis: a systematic review and meta-analysis. J Antimicrob Chemother 59, 175-183 (2007).

54. P. Farnia, F. Mohammadi, M. Mirsaedi, A. Z. Zarife, J. Tabatabee, K. Bahadori, M. Bahadori, M. R. Masjedi, A. A. Velayati, Application of oxidation-reduction assay for 
monitoring treatment of patients with pulmonary tuberculosis. J Clin Microbiol 42, 33243325 (2004).

55. T. Jirapanjawat, B. Ney, M. C. Taylor, A. C. Warden, S. Afroze, R. J. Russell, B. M. Lee, C. J. Jackson, J. G. Oakeshott, G. Pandey, C. Greening, The redox cofactor F420 protects mycobacteria from diverse antimicrobial compounds and mediates a reductive detoxification system. Applied and environmental microbiology, (2016).

56. M. S. Jang, Y. M. Lee, C. H. Kim, J. H. Lee, D. W. Kang, S. J. Kim, Y. C. Lee, Triphenylmethane reductase from Citrobacter sp. strain KCTC 18061P: purification, characterization, gene cloning, and overexpression of a functional protein in Escherichia coli. Applied and environmental microbiology 71, 7955-7960 (2005).

57. V. Fessard, T. Godard, S. Huet, A. Mourot, J. M. Poul, Mutagenicity of malachite green and leucomalachite green in in vitro tests. J Appl Toxicol 19, 421-430 (1999).

58. M. Gurumurthy, M. Rao, T. Mukherjee, S. P. Rao, H. I. Boshoff, T. Dick, C. E. Barry, 3rd, U. H. Manjunatha, A novel F(420) -dependent anti-oxidant mechanism protects Mycobacterium tuberculosis against oxidative stress and bactericidal agents. Molecular microbiology 87, 744-755 (2013).

59. K. H. Darwin, S. Ehrt, J. C. Gutierrez-Ramos, N. Weich, C. F. Nathan, The proteasome of Mycobacterium tuberculosis is required for resistance to nitric oxide. Science 302, 1963-1966 (2003).

TABLES

Table 1. Mutations identified in 89 individual colonies and pooled isolates of pretomanidresistant $M$. tuberculosis selected in BALB/C mice 
bioRxiv preprint doi: https://doi.org/10.1101/457754; this version posted October 31, 2018. The copyright holder for this preprint (which was not certified by peer review) is the author/funder. All rights reserved. No reuse allowed without permission.

\begin{tabular}{|c|c|c|c|c|c|c|c|c|}
\hline \multirow{2}{*}{$\begin{array}{c}\text { Pretomanid } \\
\text { treatment }\end{array}$} & \multirow[b]{2}{*}{ Mouse } & \multirow{2}{*}{$\begin{array}{c}\text { No. of } \\
\text { single isolates }\end{array}$} & \multicolumn{6}{|c|}{ Gene name, amino acid (aa) length and no. of unique mutations selected $(n=54)$} \\
\hline & & & RV3261 (fbiA) & $R \vee 3262(f b i B)$ & Rv1173 (fbic) & Rv3547(ddn) & Rv0407(fgd1) & $R v 2983(\operatorname{cof} C)$ \\
\hline$(\mathrm{mg} / \mathrm{kg})$ & ID & $(n=89)$ & $331 \mathrm{aa}(\mathrm{n}=7)$ & 448aa $(n=3)$ & $856 a a(n=29)$ & 151aa $(n=5)$ & $336 \mathrm{aa}(\mathrm{n}=2)$ & 214aa $(n=8)$ \\
\hline \multirow[t]{4}{*}{0} & M-1 & 2 & & & & $-G$ in aa 38 & K9N & \\
\hline & $M-2$ & 4 & & & R133P (2); -A in aa 503 & & K9N & \\
\hline & $M-4$ & 1 & & & W690* & & & \\
\hline & $M-5$ & 4 & & & $+A$ in aa $426(4)$ & & & \\
\hline \multirow[t]{6}{*}{10} & $M-1$ & 8 & A212V & & L702R (4); C562W & $-G C$ in aa 39 & & $+C$ in aa 27 \\
\hline & $M-2$ & 2 & & & 9 bp del (aa 307-309); & & & \\
\hline & & & & & -TCCCCGATG del in aa 306-308 & & & \\
\hline & $M-3$ & 4 & & L15P & L772W; -C in aa 252 (2) & & & \\
\hline & $M-4$ & 5 & & & & & & G147C (5) \\
\hline & $M-5$ & 2 & & & $\mathrm{~A} 632 \mathrm{~V}$ & & & R25G \\
\hline \multirow[t]{5}{*}{30} & $M-1$ & 1 & & & S169P & & & \\
\hline & $M-2$ & 3 & L211P & & & W20* & & A132V \\
\hline & $M-3$ & 2 & & & R278P (2) & & & \\
\hline & M-4 & 1 & & & W155* & & & \\
\hline & $M-5$ & 3 & & & Q400*; $+\mathrm{T}$ in aa $188(2)$ & & & \\
\hline \multirow[t]{5}{*}{100} & $M-1$ & 1 & & & & & & -ATC in aa 129 \\
\hline & $M-2$ & 3 & & & K682E; Y170*; L187R & & & \\
\hline & $M-3$ & 2 & & W397R; L173P & & & & \\
\hline & $M-4$ & 2 & & & F744S & $-C$ in aa 93 & & \\
\hline & $M-5$ & 4 & S219G & & M776Т (3) & & & \\
\hline \multirow[t]{5}{*}{300} & $M-1$ & 7 & & & & & & R25S (7) \\
\hline & $M-2$ & 4 & Q27 $7^{*}(3) ;-G$ in aa 248 & & & & & \\
\hline & $M-3$ & 4 & & & H190N; Q20*; -GC in aa 846 (2) & & & \\
\hline & M-4 & 4 & & & T707M; Т301A; -C in aa 247 & 9345 bp del (Rß3540-Rß3550) & & \\
\hline & $M-5$ & 7 & W79* $(6)$ & & & & & A198P \\
\hline \multirow[t]{4}{*}{600} & $M-1$ & 1 & & & N336K & & & \\
\hline & $M-2$ & 5 & & & $R 500^{*}(5)$ & & & \\
\hline & $M-3$ & 1 & D49G & & & & & \\
\hline & M-4 & 2 & & & & & & $\mathrm{C} 152 \mathrm{R}(2)$ \\
\hline
\end{tabular}

Note: * : stop codon: + or ins: insertion; - or del: deletion; no. in parenthesis next to a mutation: no. of isolates with the same mutation. 
Table 2. Mutations identified in 64 individual colonies and pooled isolates of nitrioimidazole-resistant M. tuberculosis selected in C3HeB/FeJ mice

\begin{tabular}{|c|c|c|c|c|c|c|c|c|}
\hline \multirow{3}{*}{$\begin{array}{c}\text { Pretomanid } \\
\text { treatment } \\
(\mathrm{mg} / \mathrm{kg})\end{array}$} & \multirow{3}{*}{\begin{tabular}{|c|} 
Mouse \\
ID
\end{tabular}} & \multirow{3}{*}{$\begin{array}{c}\text { No. of } \\
\text { single isolates } \\
(n=64)\end{array}$} & \multicolumn{6}{|c|}{ Gene name, amino acid (aa) length and no. of unique mutations selected $(n=45)$} \\
\hline & & & Rv3261 (fbiA) & Rv3262 (fbiB) & Rv1173 (fbic) & Rv3547 (ddn) & Rv0407 (fgd1) & Rv2983 (cofC) \\
\hline & & & 331 aa $(n=8)$ & 448aa $(n=1)$ & $856 a a(n=25)$ & 151aa $(n=7)$ & $336 \mathrm{aa}(\mathrm{n}=2)$ & 214aa $(n=2)$ \\
\hline \multirow[t]{4}{*}{10} & $\mathrm{M}-1$ & 4 & & & $-A$ in aa 331;G194D; W198* & & & A68E \\
\hline & $\mathrm{M}-3$ & 2 & & & $-\mathrm{C}$ in aa 20 & L49P & & \\
\hline & M-4 & 2 & & & K684T & & & Q114R \\
\hline & $\mathrm{M}-5$ & 1 & & & del 42 bp (aa 680-693) & & & \\
\hline \multirow{3}{*}{30} & $M-2$ & 5 & $-G C$ in aa 140 & & L377P (2); L377P (2, het, p) & & & \\
\hline & M-4 & 3 & & & G148D; W354R & C149Y & & \\
\hline & $M-5$ & 3 & G273D (2) & & $1 \mathrm{~kb}$ del (fbiC+PE12) & & & \\
\hline \multirow[t]{4}{*}{100} & $M-1$ & 5 & & & & IS6110 ins. in aa 131 & G191D (5) & \\
\hline & $\mathrm{M}-2$ & 1 & & & del 4 bp in aa $75-76$ & & & \\
\hline & $\mathrm{M}-3$ & 3 & $-G$ in aa $47(2)$ & & L685R & & & \\
\hline & M-4 & 5 & $+C$ in aa 125 & & A588P (2): -G in aa 52; R322L & & & \\
\hline \multirow{2}{*}{300} & M-4 & 3 & & & & R112W (2); IS6110 ins. in D108 & & \\
\hline & $M-5$ & 4 & D286A & & T273K (2) & $-G$ in aa 39 & & \\
\hline
\end{tabular}

Table 3. Distribution of mutation types and frequencies in genes associated with pretomanid resistance, by mouse strain

\begin{tabular}{|c|c|c|c|c|c|c|c|c|c|}
\hline \multirow{3}{*}{$\begin{array}{l}\text { Mouse } \\
\text { model }\end{array}$} & \multirow{3}{*}{ Type of mutation } & \multicolumn{6}{|c|}{ Gene name and amino acid (aa) length } & \multirow{3}{*}{$\begin{array}{c}\text { No. of } \\
\text { mutation } \\
\end{array}$} & \multirow{3}{*}{$\begin{array}{c}\text { Frequenc } \\
\text { of } \\
\text { mutation }\end{array}$} \\
\hline & & \multirow{2}{*}{\begin{tabular}{|c|} 
Rv3261 (fbiA) \\
331aa
\end{tabular}} & \multirow{2}{*}{$\begin{array}{c}R v 3262(f b i B) \\
\text { 448aa }\end{array}$} & \multirow{2}{*}{$\begin{array}{c}\text { Rv1173 (fbic) } \\
\text { 856aa }\end{array}$} & \multirow{2}{*}{$\begin{array}{c}R v 3547(d d n) \\
151 \mathrm{aa}\end{array}$} & \multirow{2}{*}{$\begin{array}{c}\text { Rv0407(fgd1) } \\
\text { 336aa } \\
\end{array}$} & \multirow{2}{*}{$\begin{array}{c}R v 2983(\mathrm{cofC}) \\
\text { 214aa }\end{array}$} & & \\
\hline & & & & & & & & & \\
\hline \multirow[t]{5}{*}{$\mathrm{BALB} / \mathrm{C}$} & no. of point mutation & 4 & 3 & 15 & 0 & 2 & 6 & 30 & $56 \%$ \\
\hline & no. of indels (ins+del) & 1 & 0 & 8 & 4 & 0 & 2 & 15 & $28 \%$ \\
\hline & no. of stop codon & 2 & 0 & 6 & 1 & 0 & 0 & 9 & $17 \%$ \\
\hline & no. of total mutation & 7 & 3 & 29 & 5 & 2 & 8 & 54 & $100 \%$ \\
\hline & frequency of mutation & $13 \%$ & $6 \%$ & $54 \%$ & $9 \%$ & $4 \%$ & $15 \%$ & $100 \%$ & \\
\hline \multirow[t]{5}{*}{$\mathrm{C} 3 \mathrm{HeB} / \mathrm{FeJ}$} & no of point mutation & 5 & 0 & 12 & 3 & 1 & 2 & 23 & $51 \%$ \\
\hline & no of indels (ins+del) & 3 & 1 & 12 & 3 & 1 & 0 & 20 & $44 \%$ \\
\hline & no. of stop codon & 0 & 0 & 1 & 1 & 0 & 0 & 2 & $4 \%$ \\
\hline & no. of total mutation & 8 & 1 & 25 & 7 & 2 & 2 & 45 & $100 \%$ \\
\hline & frequency of mutation & $18 \%$ & $2 \%$ & $56 \%$ & $16 \%$ & $4 \%$ & $4 \%$ & $100 \%$ & \\
\hline
\end{tabular}

Note: ins: insertion; del: deletion. 


\section{FIGURES}

A.

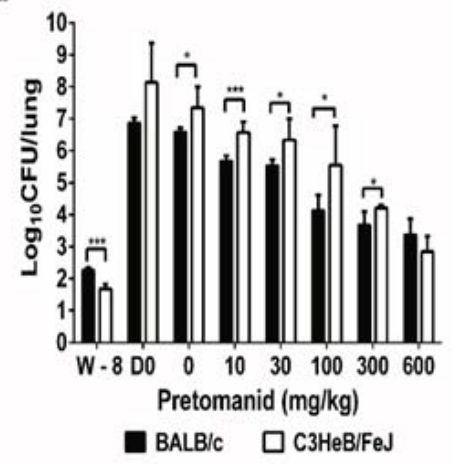

B.

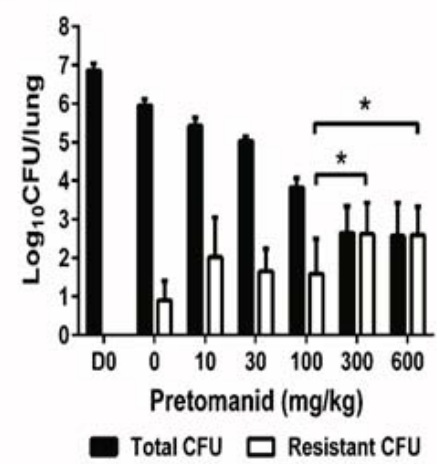

C.

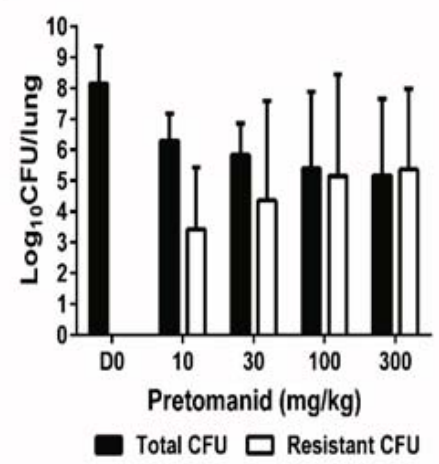

Fig. 1. Selective amplification of spontaneous pretomanid-resistant mutants during pretomanid monotherapy in mice is dose-dependent and is more pronounced in C3HeB/FeJ mice. After aerosol infection with M. tuberculosis H37Rv, BALB/c and C3HeB/FeJ mice were treated with a range of doses of pretomanid for 8 weeks and sacrificed at different time points before and after treatment for lung CFU counts. A. Mean ( \pm S.D.) total lung CFU counts on the day after infection (W-8), on the day of treatment initiation (D0), and after 3 weeks of treatment with the indicated pretomanid dose (in $\mathrm{mg} / \mathrm{kg}$ body weight). Dose-dependent bactericidal activity was observed in both strains; B. Mean ( \pm S.D.) total and PMD-resistant lung CFU counts in BALB/c mice on day 0 and after 8 weeks of treatment with the indicated pretomanid dose. Dosedependent bactericidal activity and selection of PMD-resistant bacteria was observed, with the resistant population overtaking the susceptible population at doses $\geq 300 \mathrm{mg} / \mathrm{kg}$; C. Mean ( \pm S.D.) total and PMD-resistant lung CFU counts in C3HeB/FeJ mice on day 0 and after 8 weeks of treatment with the indicated pretomanid dose. Dose-dependent bactericidal activity and selection of PMD-resistant bacteria was observed, with the resistant population overtaking the susceptible population at doses $\geq 30 \mathrm{mg} / \mathrm{kg} .{ }^{\star} p<0.05,{ }^{\star \star \star} p<0.001$ 


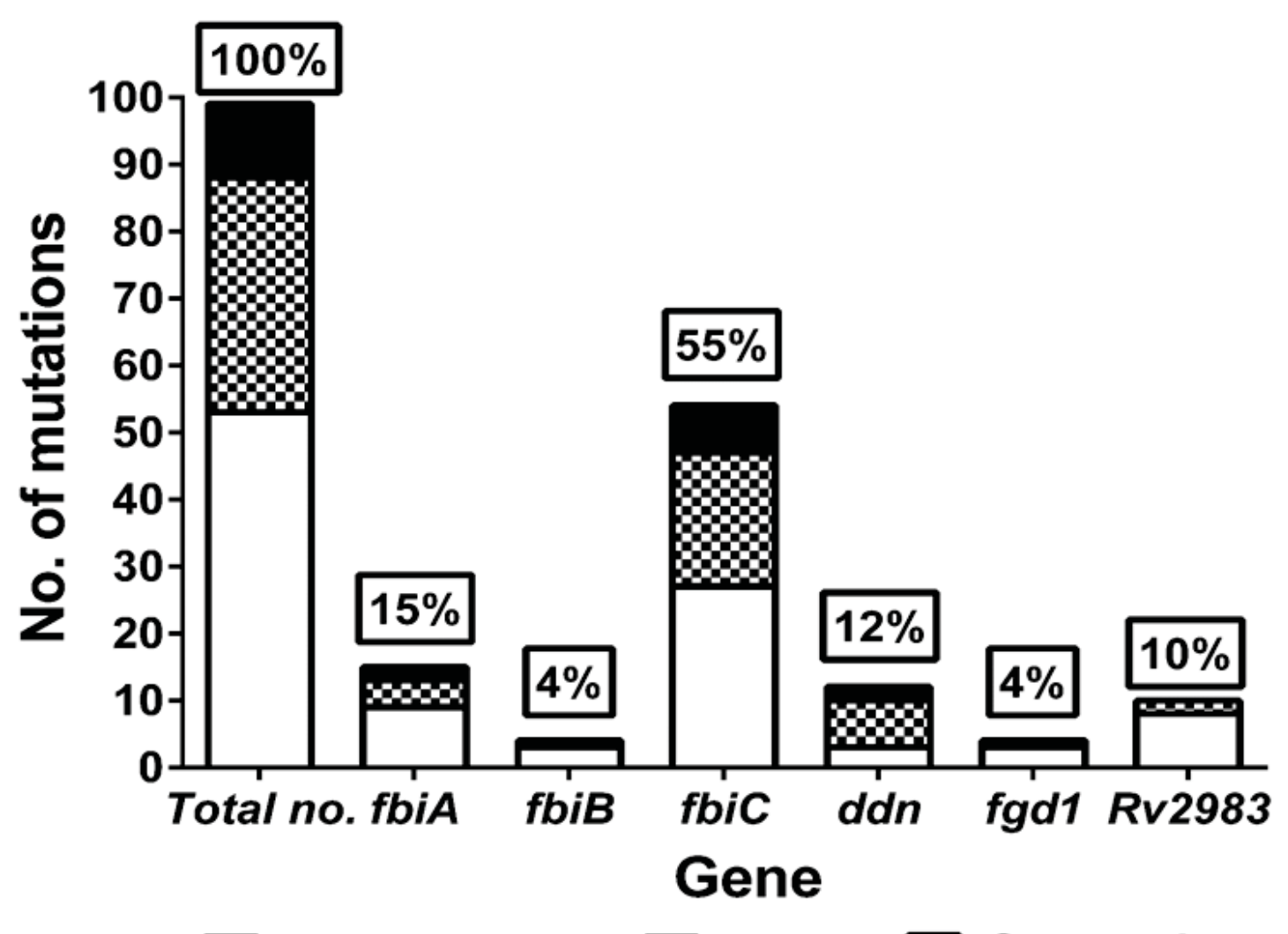

\section{$\square$ Point mutation $\mathbb{B}$ Indels $\square$ Stop codon}

Fig. 2. Overall mutation frequencies of genes associated with pretomanid resistance. WGS was performed with 136 pretomanid-resistant colonies and 25 colony pools picked from 47 individual mice harboring pretomanid-resistant CFU after 8 weeks of treatment and identified 99 unique mutations in these 6 genes. Mutations in fbic (55\%) were the predominant cause of pretomanid resistance. For the other 5 genes, the rank order by mutation frequency was fbiA (15\%), ddn (12\%), Rv2983 (10\%), fgd1 (4\%) and fbiB (4\%). 
A.

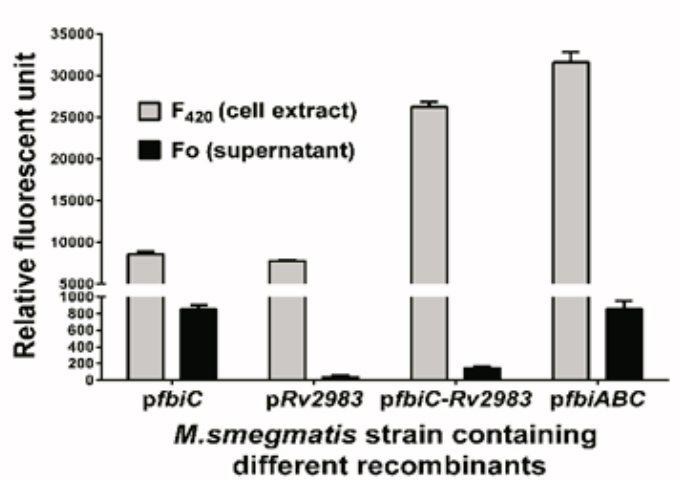

C.

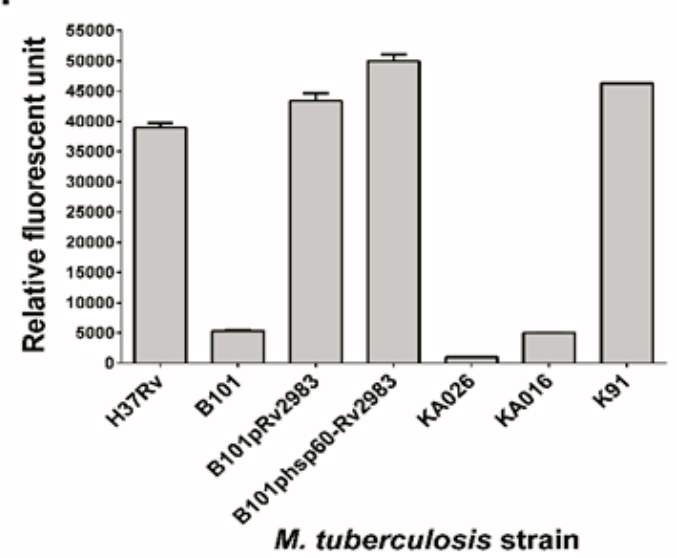

B.

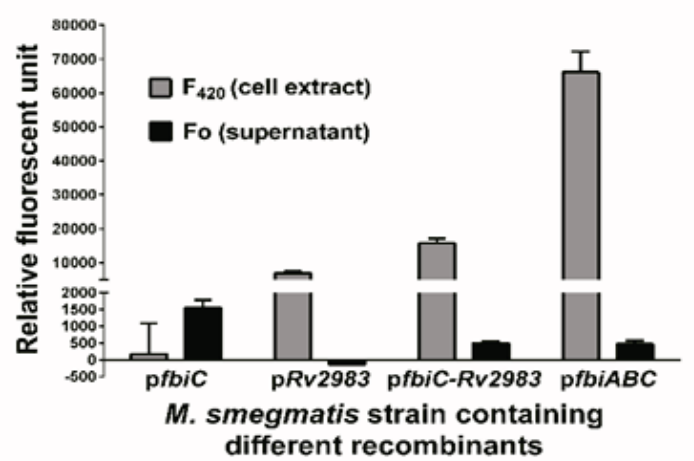

D.

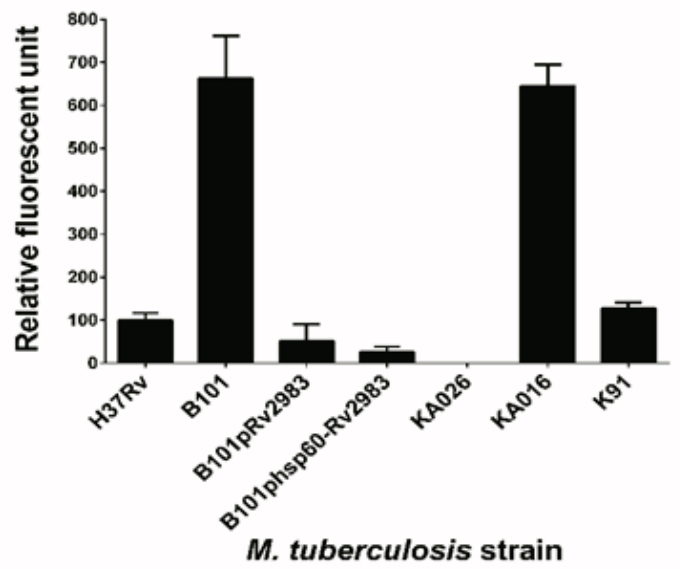

E.

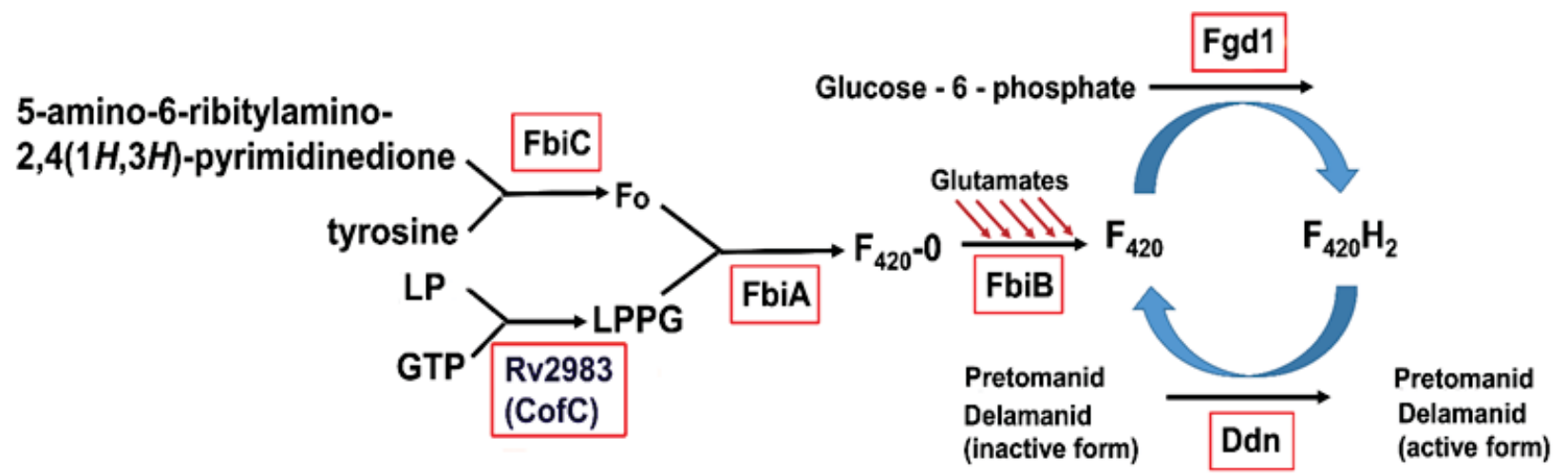

Fig. 3. Rv2983 is required for efficient $F_{420}$ synthesis from Fo. $F_{420}$ and Fo content was measured in $M$. smegmatis strains harboring different recombinants relative to the control strain containing the empty vector pYUBDuet after $6(\mathrm{~A})$ and $26(\mathrm{~B})$ hours of $1 \mathrm{mM}$ IPTG induction; $\mathrm{F}_{420}$ (C) and Fo (D) content was measured in Rv2983 mutant strains of M. tuberculosis and control 
strains including B101 ( $\Delta R v 2983, \mathrm{~A} 198 \mathrm{P}), \mathrm{KA} 016$ ( $\Delta$ Rv2983, Q114R), H37Rv (wild-type), B101 complemented strain (pMH94-Rv2983), B101 complemented strain (pMH94-hsp60-Rv2983), KA026 ( $\Delta$ fbiC, IS6110 insertion in 85-bp upstream of $f b i C)$, and K91 ( $\Delta d d n$, IS6110 insertion in aa D108), after growth in $7 \mathrm{H} 9$ broth for 6 days. Schematic diagram (E) of proposed nitroimidazole activation pathway showing Rv2983 as putative CofC catalyzing LPPG biosynthesis. Fo, 7,8didemethyl-8-hydroxy-5-deazariboflavin; LP, 2-phospho-L-lactate; GTP, guanosine triphosphate; LPPG, L-lactyl-2-diphospho-5'-guanosine. 
A.

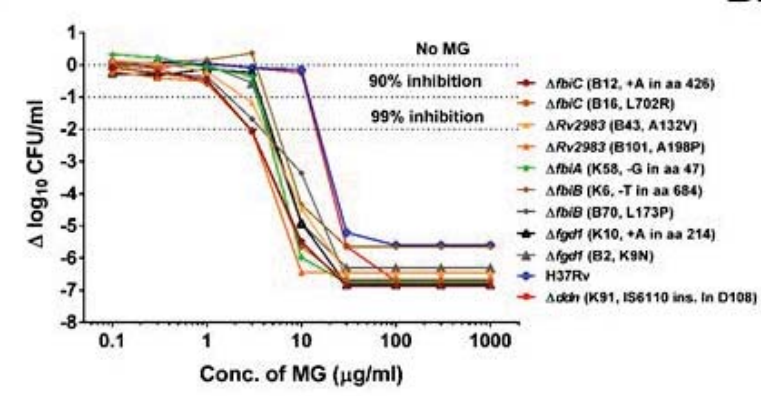

C.

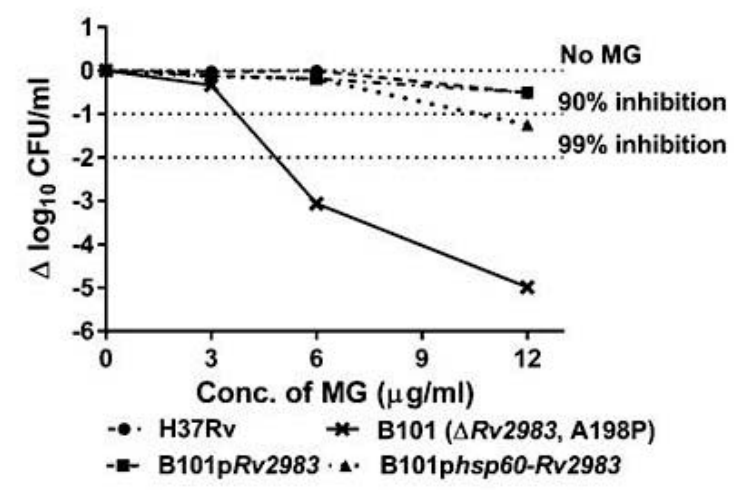

B.

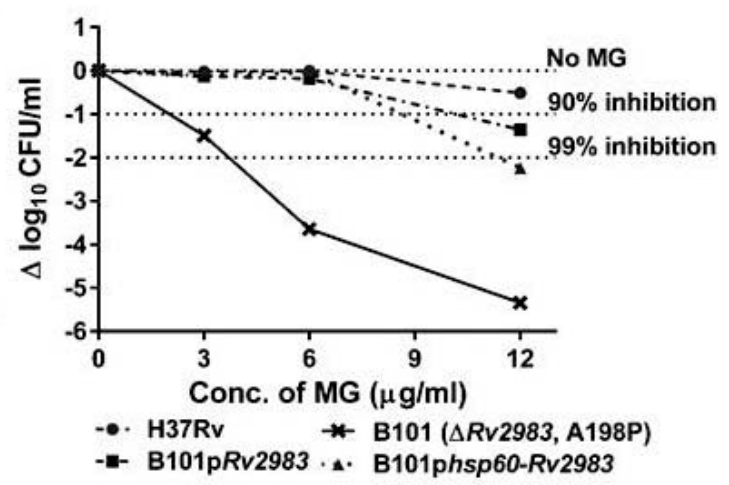

D.

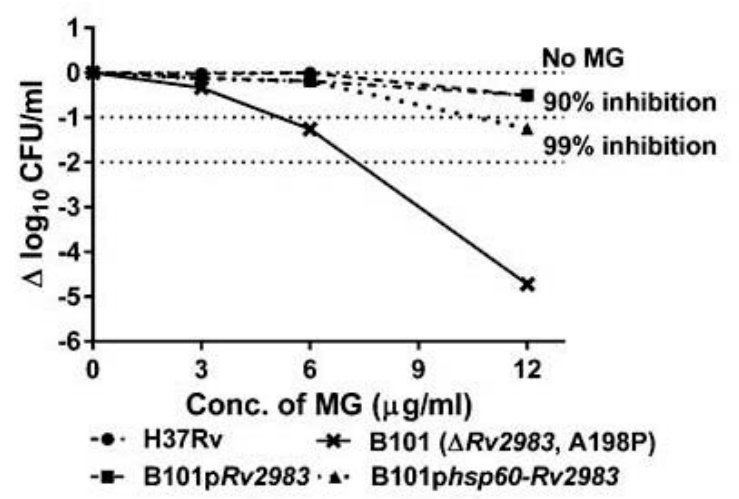

Fig. 4. $F_{420} \mathrm{H}_{2}$-deficient pretomanid-resistant mutants of $M$. tuberculosis are more susceptible to growth inhibition by malachite green. A. Growth of wild-type M. tuberculosis on $7 \mathrm{H} 9$ agar is inhibited by malachite green (MG) in a concentration-dependent manner. $\mathrm{F}_{420} \mathrm{H}_{2-}$ deficient, pretomanid-resistant M. tuberculosis mutants (fbiA-C, fgd1, Rv2983) are inhibited at lower MG concentrations relative to the wild type and the $\mathrm{F}_{420} \mathrm{H}_{2}$-sufficient, pretomanid-resistant ddn mutant. B-D. Complementation of the B101 mutant with wild-type Rv2983 restores tolerance to $M G$ and the proportional recovery of the mutant on $6 \mu \mathrm{g} / \mathrm{ml}$ of MG increases as the duration of incubation increases from 28 (B), to 35 (C) to 49 (D) days of incubation. The proportional recovery of the mutant on plates containing $12 \mu \mathrm{g} / \mathrm{ml}$ of MG does not increase with time. 


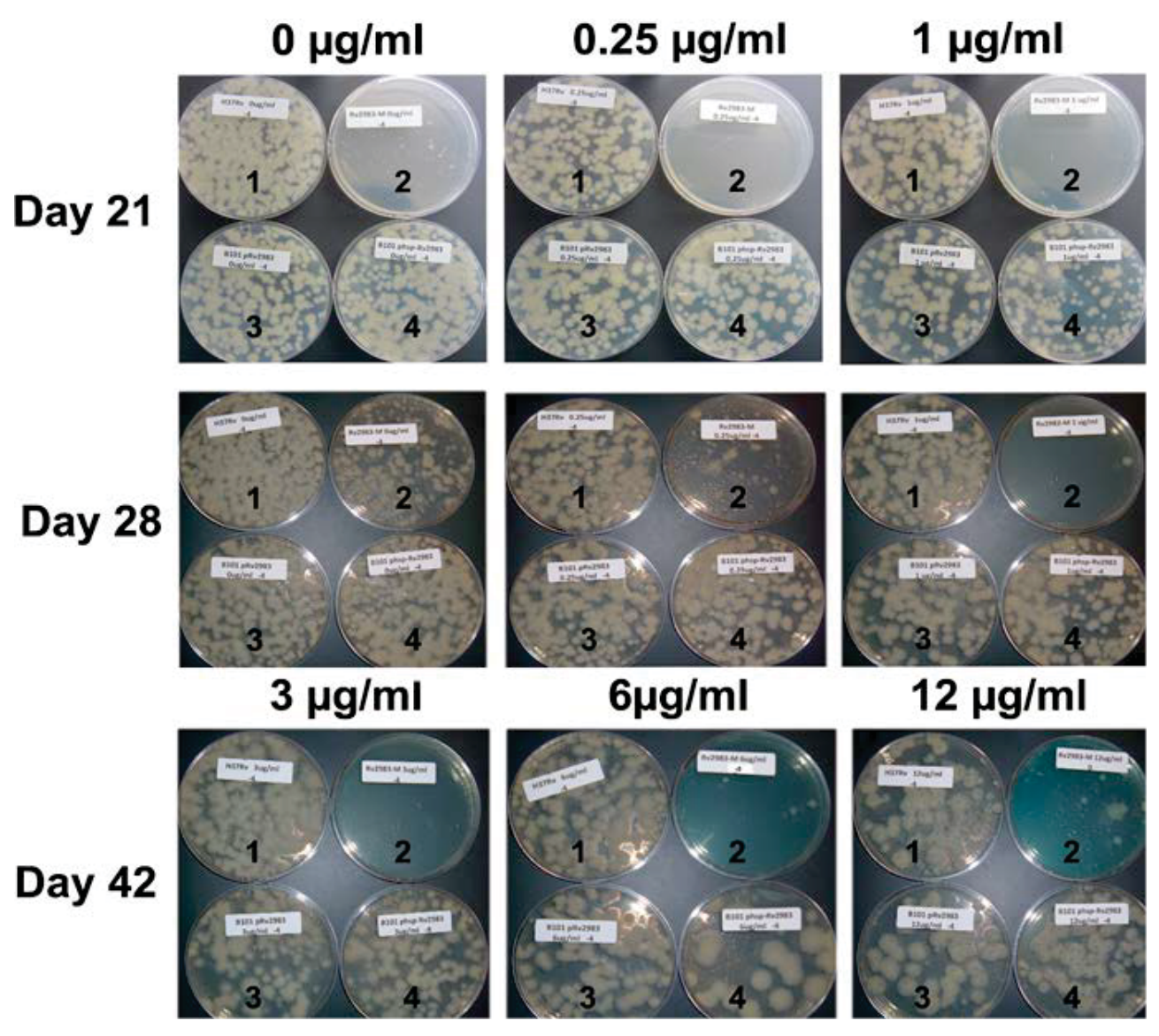

Fig. 5. Mutation of Rv2983 causes growth inhibition and defective decolorization of malachite green. Aliquots $(100 \mu \mathrm{l})$ of $M$. tuberculosis cultures (1, H37Rv wild type; 2, Rv2983 mutant B101; 3, B101 mutant complemented with Rv2983 behind native promoter; 4, B101 mutant complemented with Rv2983 behind hsp60 promoter) were spread on $7 \mathrm{H} 9$ agar plates containing increasing concentrations of malachite green (MG) after serial 10-fold dilutions. Colony size and MG decolorization are depicted after different incubation times. Numbers on plate labels (e.g., $0,3,4,5)$ indicate the number of 10 -fold dilutions performed before an aliquot was plated. Although the B101 mutant grows more slowly on plates without MG, growth is further slowed in the presence of $\geq 0.25 \mu \mathrm{g} / \mathrm{ml}$ of $M G$ and the proportion of CFU recovered declines with increasing MG concentrations above $0.25 \mu \mathrm{g} / \mathrm{ml}$. For plates containing 6 and $12 \mu \mathrm{g} / \mathrm{ml}$ of $\mathrm{MG}$, plates receiving more-concentrated aliquots of the B101 mutant culture are shown to demonstrate the markedly reduced recovery of the mutant at these MG concentrations. 
A.

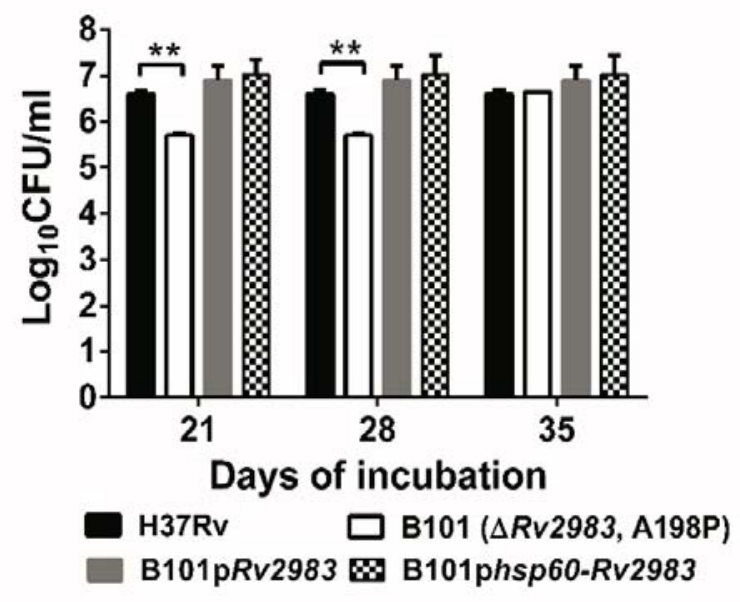

B.

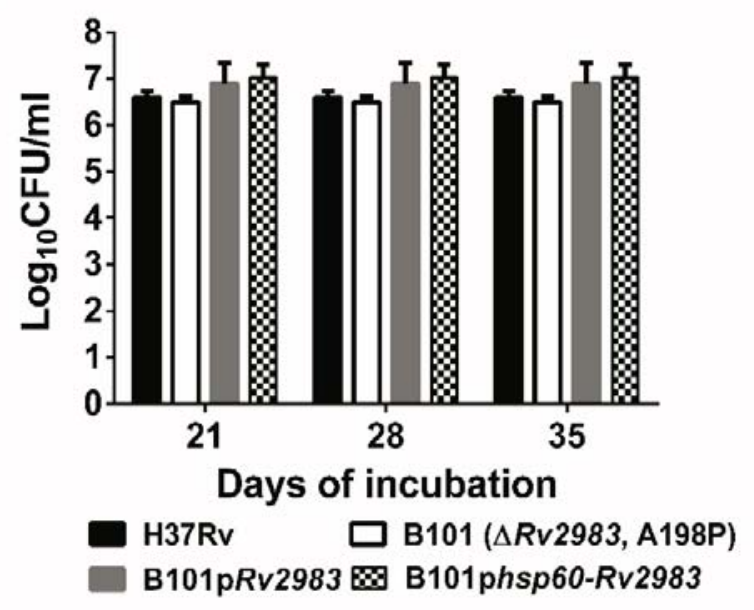

C.

(a)

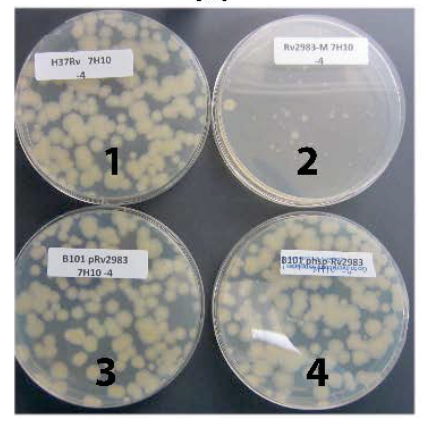

D.

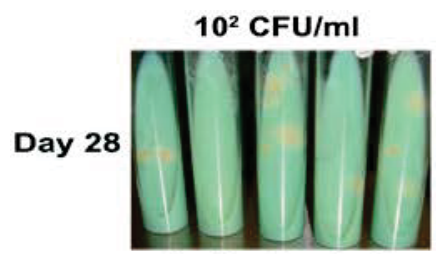

$\begin{array}{lllll}12 & 2 & 4\end{array}$

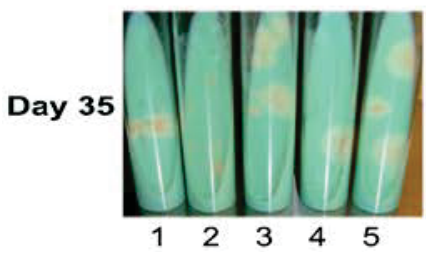

(b)

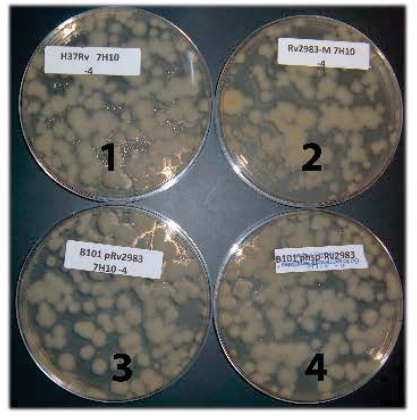

(c)

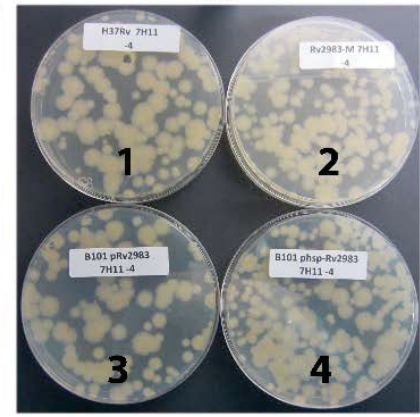

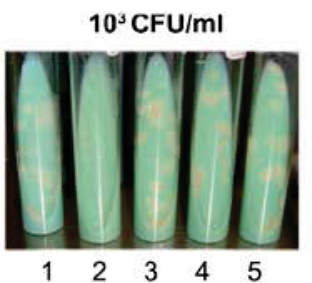

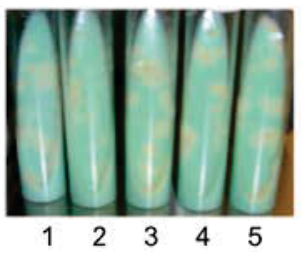

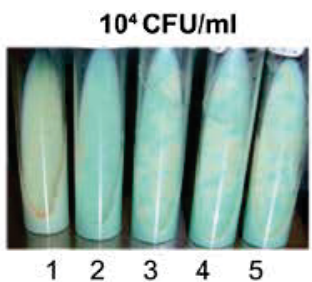

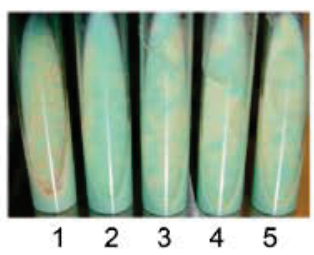

$10^{5} \mathrm{CFU} / \mathrm{ml}$

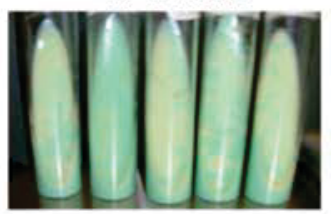

12345

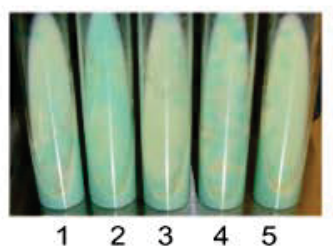

$10^{6} \mathrm{CFU} / \mathrm{ml}$
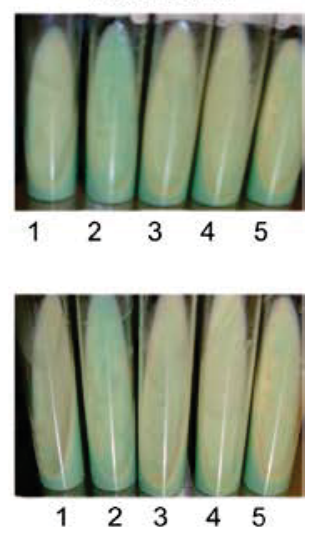
Fig. 6. Mutation of Rv2983 causes growth inhibition on commercial 7H10 agar and LJ slants, but not on commercial 7H11 agar. Aliquots of $M$. tuberculosis cultures were spread on various solid media purchased commercially after serial 10 -fold dilutions. A-B. Mean CFU counts on $7 \mathrm{H} 10(\mathrm{~A})$ and $7 \mathrm{H} 11$ (B) agar plates; C. Colonies on 7H10 agar plates after 21 (a) and 35 (b) days of incubation, and on 7H11 agar plates after 21 (c) days of incubation; D. Colonies on LJ slants inoculated with serially diluted aliquots after 28 and 35 days of incubation. 1: H37Rv wild type; 2: B101 mutant ( $\Delta R v 2983, \mathrm{~A} 198 \mathrm{P})$; 3: B101 mutant complemented with Rv2983 behind the native promoter; 4: B101 mutant complemented with Rv2983 behind the hsp60 promoter; 5. K91 mutant ( $\Delta d d n$, IS6110 ins in D108). 


\section{SUPPLEMENTARY MATERIALS}

Table S1. List of the primers used in the study

Table S2. WGS results for 136 individual pretomanid-resistant colonies selected with different doses of pretomanid in M. tuberculosis-infected BALB/C and C3HeB/FeJ mice

Table S3. WGS results for 25 pooled pretomanid-resistant isolates selected with different doses of pretomanid in M. tuberculosis-infected BALB/c and C3HeB/FeJ mice

Table S4. Distribution of overall mutation types and frequencies in genes associated with pretomanid resistance

Table S5. Pretomanid MICs against selected pretomanid-resistant M. tuberculosis mutants

Figure S1. Complementation of B101 mutant with Rv2983. A. Schematic diagram of genomic DNA of M. tuberculosis strains after digestion with restriction enzyme Acc65I; B. Result of southern blot confirmed expected DNA fragments after Acc65I digestion using DIG-labeled Rv2983 probe (H37Rv: 6.3 kb; Rv2983 mutant: 6.3 kb; complemented strains: 6.3 and 3.5 kb).

Figure S2. Expression of Rv2983 and other genes involved in nitroimidazole activation. A. Expression of Rv2983 and other genes involved in nitroimidazole activation is higher in the Rv2983 mutant B101 relative to the wild-type H37Rv after 4 days of incubation in 7H9 broth; B. fbiC expression is dramatically lower in the fbiC mutant KA026 relative to the wild-type after 2 days of incubation in $7 \mathrm{H9}$ broth; C. A faint band representing the 937-bp fbiC DNA fragment is evident in the sample from the KA026 mutant (lane 2) relative to that in H37Rv (lane 3). Lane 1 is the 1-kb DNA marker.

Figure S3. Complementation of the B101 mutant with wild-type Rv2983 restores tolerance to MG. The proportional recovery of the mutant on $6 \mu \mathrm{g} / \mathrm{ml}$ of MG increases with the volume of culture plated and the duration of incubation: 28-day incubation of $500 \mu \mathrm{l}(\mathrm{A})$ aliquots/plate; 35-day incubation of $500 \mu \mathrm{l}(\mathrm{B})$ aliquots/plate. 


\section{SUPPLEMENTARY MATERIALS}

Table S1. List of the primers used in the study

\begin{tabular}{|c|c|c|}
\hline Primer name & Primer sequence & Purpose of amplification \\
\hline Rv2983-1F & GCTCGGTACCCGGGGATCCTCCCAAGTGCCTCCTCGGC & Rv2983 OFR and flanking sequences \\
\hline Rv2983-1R & GGTGCCCTTGGTGGTCGACTGTTCGCCGTTGGTCTGCC & \\
\hline hsp60-F & GCTCGGTACCCGGGGATCCTTCGGCCATGACAAGAATCTG & hsp60 promoter \\
\hline hsp60-R & TGCCGCTCATGCATGTTTGGGCGCATCC & \\
\hline Rv2983 - 2F & CCAAACATGCATGAGCGGCACACCGGAC & Rv2983 ORF \\
\hline Rv2983-2R & GGTGCCCTTGGTGGTCGACTTCAACGATGTGCGACCGC & \\
\hline Rv2983-3F & GACTCGCGAGAACGTGGT & Rv2983 DNA probe \\
\hline Rv2983-3R & CCAGGCTCCTGTCAGCTC & \\
\hline Rv2983-4F & GTATAAGAAGGAGATATACAATGAGCGGCACACCGGAC & Rv2983 ORF \\
\hline Rv2983 - 4R & TGGCAGCAGCCTAGGTTAATTCAACGATGTGCGACCGC & \\
\hline RV2983-RT-F & CGATTTGCCGGCATTACAGA & expression of Rv2983 \\
\hline Rv2983-RT-R & CGAACGCACACAGTACCG & \\
\hline fbiC-RT-F & GTCCATTCCGTTTACCACCG & expression of $\mathrm{fbiC}$ \\
\hline fbiC-RT-R & CGGAAGTTCTGCACGATCAC & \\
\hline fbiA-RT-1F & CCAGTTTGCTGCCAATTCTG & expression of fbiA \\
\hline fbiA-RT-1R & ACATGCAGGTGTCCAGATCC & \\
\hline fbiB-RT-F & GGATGAAAGACAAGTGGCGG & expression of $f b i B$ \\
\hline fbiB-RT-R & ACTTCGGGTGCGTCATAGAG & \\
\hline fgd1-RT-F & GTACCCGAACCGTGTTTTCC & expression of $f g d 1$ \\
\hline fgd1-RT-R & CATTAGCCCCACCGATTCAC & \\
\hline $\operatorname{sig} A-\mathrm{F}$ & CTACGCTACGTGGTGGATTC & expression of $\operatorname{sig} A$ \\
\hline $\operatorname{sig} A-\mathrm{R}$ & GGTGATGTCCATGTCTTTGG & \\
\hline fbiC-5-7_F & GCAAGGTGTTTATCCCGGTC & fbiC DNA fragment \\
\hline fbiC-5-7_R & TGTACGTATTTGGGTTGCGC & \\
\hline
\end{tabular}

Table S2. WGS results for 136 individual pretomanid-resistant colonies selected with different doses of pretomanid in M. tuberculosis-infected BALB/c and C3HeB/FeJ mice 
bioRxiv preprint doi: https://doi.org/10.1101/457754; this version posted October 31, 2018. The copyright holder for this preprint (which was not certified by peer review) is the author/funder. All rights reserved. No reuse allowed without permission.

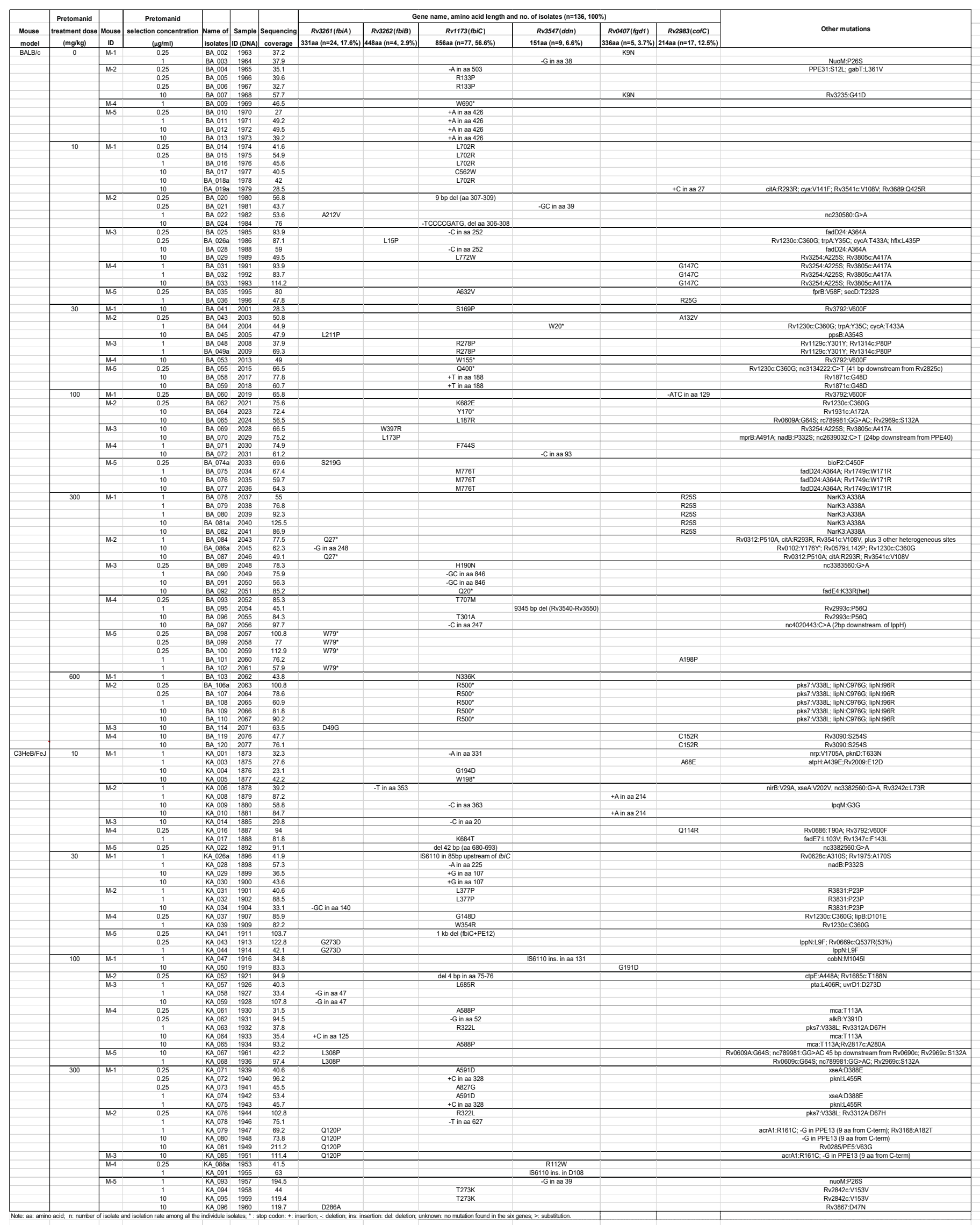


Table S3. WGS results for 25 pooled pretomanid-resistant isolates selected with different doses of pretomanid in M. tuberculosis-infected BALB/c and C3HeB/FeJ mice

\begin{tabular}{|c|c|c|c|c|c|c|c|c|c|c|c|c|c|}
\hline \multirow[b]{2}{*}{ Mouse } & \multirow{2}{*}{\begin{tabular}{|l} 
Pretomanid \\
treatment dose
\end{tabular}} & \multirow[b]{2}{*}{ Mouse } & \multirow{2}{*}{\begin{tabular}{c|} 
Pretomanid \\
selection concentration
\end{tabular}} & \multirow{2}{*}{$\begin{array}{l}\text { Name of } \\
\text { pooled }\end{array}$} & \multirow[b]{2}{*}{ Sample } & \multirow[b]{2}{*}{ Sequencing } & \multicolumn{6}{|c|}{ Gene name and amino acid (aa) length } & \multirow{3}{*}{ Other mutations } \\
\hline & & & & & & & Ru3261 (thia) & RU3262 (tbiB) & Rn173(thic) & Ris547(ddn) & RW0407(fgd1) & RV2983(CofC) & \\
\hline model & (mglkg) & ID & $(\mu \mathrm{g} / \mathrm{mll})$ & isolates & ID (DNA) & coverage & 331aa & 448aa & 856aa & 151aa & 336аa & 214aa & \\
\hline \multirow[t]{8}{*}{ Balbc } & 10 & M-4 & 1 & P1 & 2084 & 103.4 & & & & & & G147C & Rv3254:A225S; Rv3805C:A417A \\
\hline & & & 10 & P2 & 2085 & 140.3 & & & & & & G147C & Rv3254:A2255; Rv3805c:A417A \\
\hline & 300 & M-1 & 1 & P3 & 2086 & 123.1 & & & & & & R25S & narK3:A338A(het) \\
\hline & & & 10 & P4 & 2087 & 74.5 & & & & & & R25S(het) & narK3:A338A(het) \\
\hline & & M.2 & 10 & P6 & 2089 & 44.6 & Q27 (het) & & & & & & Ru35411:V108V; RV0312:P510A(het); iti:R2938R(het) \\
\hline & & M.5 & 0.25 & P7 & 2090 & 44.6 & W79* & & & & & & \\
\hline & & & 1 & P8 & 2091 & 67.8 & W79* & & & & & & \\
\hline & 600 & M.5 & 1 & $\mathrm{Pg}$ & 2092 & 55.3 & & & & & & & Rv2310:E94A; Rv3792:V600F(het); proS:A524E(het) \\
\hline \multirow[t]{17}{*}{ C3HBBFFJ } & 10 & M-1 & 10 & P11 & 2094 & 39.5 & & & & & & & nrp:V1705A; pknD:T633N \\
\hline & & M.3 & 1 & P12 & 2095 & 34.4 & & & & LA9P(het) & & & \\
\hline & 30 & M-1 & 1 & P13 & 2096 & 65.9 & & & & W27t/het) & & & Rv0628C:A310S(het); Rv1975:A170S(het) \\
\hline & & & 10 & P14 & 2097 & 66.2 & & & & & & & Rv0628C:A310S(het); Rv1975:A170S(het) \\
\hline & & M.2 & 1 & P15 & 2098 & 35.5 & & & L377P(het) & & & & Rv3833:P23P \\
\hline & & & 10 & P16 & 2099 & 53.1 & & & L377P(het) & & & & RV3831:P23P \\
\hline & & M.4 & 0.25 & P17 & 2100 & 53 & & & & C149y(het) & & & Rv1230C:C360GGhet); ureG:L58_(het); Rv2567:A728A(het); Rv3229G:T133A(het); nadB:P322S|hett; Rv3447c:het \\
\hline & & & 1 & P18 & 2101 & 67.7 & & & & & & & Rv1230C:C360G(het); ureG:L58L(het); Rv2567:A728A(het); Rv3229c:T133A(het);nadB:P3225Shet) \\
\hline & 100 & M-1 & 0.25 & P19 & 2102 & 73 & & & & & G1910(het) & & cobN:M1045(het); groEl:G171G(het) \\
\hline & & & 1 & P20 & 2103 & 33.8 & & & & & G191D(het) & & cobN:M1045|(het); groEl:G171GGhet;; Rv0987:indel? \\
\hline & & & 10 & P21 & 2104 & 75 & & & & & G1910(het) & & groEl:G171G(het) \\
\hline & & M-4 & 1 & P24 & 2107 & 48.6 & & & & & & & \\
\hline & 300 & M-1 & 1 & P26 & 2108 & 44.4 & & & & & & & pkn:LL455R(het) \\
\hline & & M-3 & 1 & P27 & 2109 & 30.8 & Q120P(het) & & & & & & \\
\hline & & M-4 & 0.25 & P28 & 2110 & 93.4 & & & & R112W(het) & & & \\
\hline & & & 1 & P29 & 2111 & 142.9 & & & & & & & \\
\hline & & M.5 & 10 & P31 & 2113 & 139.5 & & & & & & & \\
\hline \multicolumn{14}{|c|}{ Note: : : stop codon; het: heterogeneous. } \\
\hline
\end{tabular}

Table S4. Distribution of overall mutation types and frequencies in genes associated with pretomanid resistance

\begin{tabular}{|c|c|c|c|c|c|c|c|c|}
\hline \multirow{3}{*}{ Type of mutation } & \multicolumn{6}{|c|}{ Gene name and amino acid (aa) length } & \multirow[b]{2}{*}{ No. of } & \multirow{2}{*}{$\begin{array}{c}\text { Frequency } \\
\text { of }\end{array}$} \\
\hline & Rv3261 (fbiA) & Rv3262 (fbiB) & Rv1173(fbic) & Rv3547(ddn) & Rv0407 (fgd1) & $R v 2983(\operatorname{cofC})$ & & \\
\hline & 331aa & 448aa & 856aa & 151aa & 336aa & 214aa & mutation & mutation \\
\hline no. of point mutation & 9 & 3 & 27 & 3 & 3 & 8 & 53 & $54 \%$ \\
\hline no. of indels (ins+del) & 4 & 1 & 20 & 7 & 1 & 2 & 35 & $35 \%$ \\
\hline no. of stop codon & 2 & 0 & 7 & 2 & 0 & 0 & 11 & $11 \%$ \\
\hline no. of total mutation & 15 & 4 & 54 & 12 & 4 & 10 & 99 & $100 \%$ \\
\hline frequency of mutation & $15 \%$ & $4 \%$ & $55 \%$ & $12 \%$ & $4 \%$ & $10 \%$ & $100 \%$ & \\
\hline
\end{tabular}

Note: ins: insertion; del: deletion. 
Table S5. Pretomanid MICs against selected pretomanid-resistant M. tuberculosis mutants

\begin{tabular}{|c|c|c|c|c|c|c|c|}
\hline Mouse & Isolate & Pretomanid treatment & Pretomanid selection & Gene & Mutation & Agar-based MIC & Broth-based MIC \\
\hline \multirow[t]{2}{*}{ model } & & dose $(\mathrm{mg} / \mathrm{kg})$ & concentration $(\mu \mathrm{g} / \mathrm{ml})$ & & & $(\mu \mathrm{g} / \mathrm{ml})$ & $(\mu \mathrm{g} / \mathrm{ml})$ \\
\hline & H37Rv parent & & & & wild-type & 0.06 & 0.25 \\
\hline \multirow[t]{3}{*}{ BALB/C } & BA_002 & 0 & 0.25 & fgd1 & K9N & $>32$ & \\
\hline & BA_016 & 10 & 1 & fbic & L702R & $>32$ & \\
\hline & BA_101 & 300 & 1 & Rv2983 & A198P & 32 & \\
\hline \multirow[t]{5}{*}{$\mathrm{C} 3 \mathrm{HeB} / \mathrm{FeJ}$} & KA_006 & 10 & 1 & $f b i B$ & del of T684 & 8 & \\
\hline & KA_058 & 100 & 1 & fbiA & del of G47 & $>32$ & \\
\hline & KA_091 & 300 & 1 & $d d n$ & D108 (IS6110 ins) & 32 & \\
\hline & KA-016 & 10 & 0.25 & Rv2983 & Q114R & & $>32$ \\
\hline & KA-026a & 30 & 1 & fbic & IS6110 ins. in 85bp upstream of fbiC & & $>32$ \\
\hline
\end{tabular}


A.

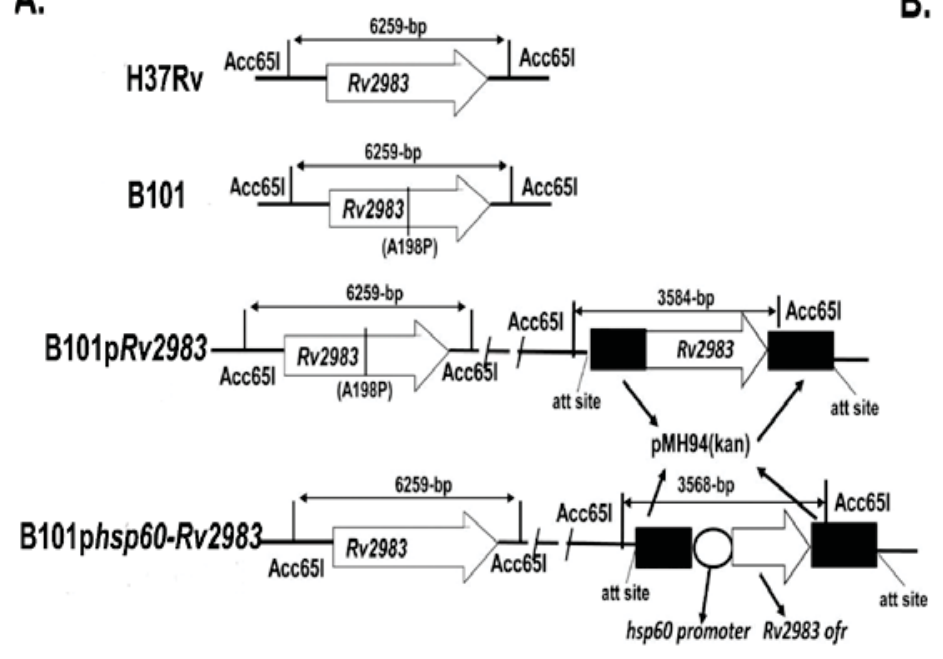

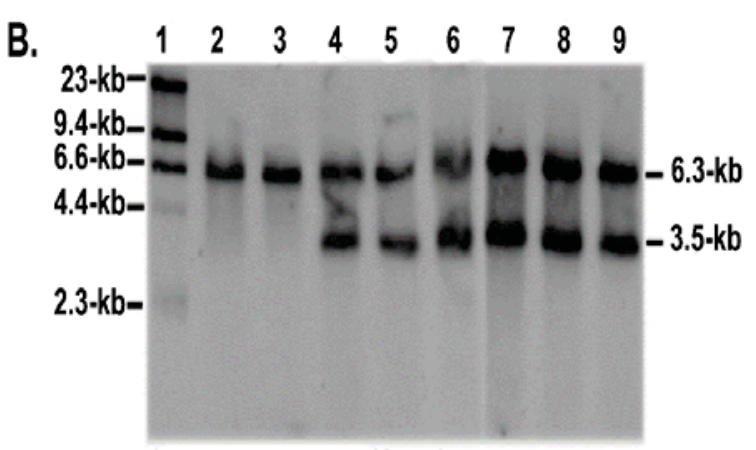

lane 1. Dig-labeled DNA marker; lane 2. H37Rv lane 3. B101 lane 4-6. B101pRv2983 candidates lane 7-9. B101phsp60-Rv2983 candidates

Figure S1. Complementation of B101 mutant with Rv2983. A. Schematic diagram of genomic DNA of M. tuberculosis strains after digestion with restriction enzyme Acc65I; B. Result of southern blot confirmed expected DNA fragments after Acc65I digestion using DIG-labeled Rv2983 probe (H37Rv: $6.3 \mathrm{~kb}$; Rv2983 mutant: $6.3 \mathrm{~kb}$; complemented strains: 6.3 and $3.5 \mathrm{~kb}$ ). 

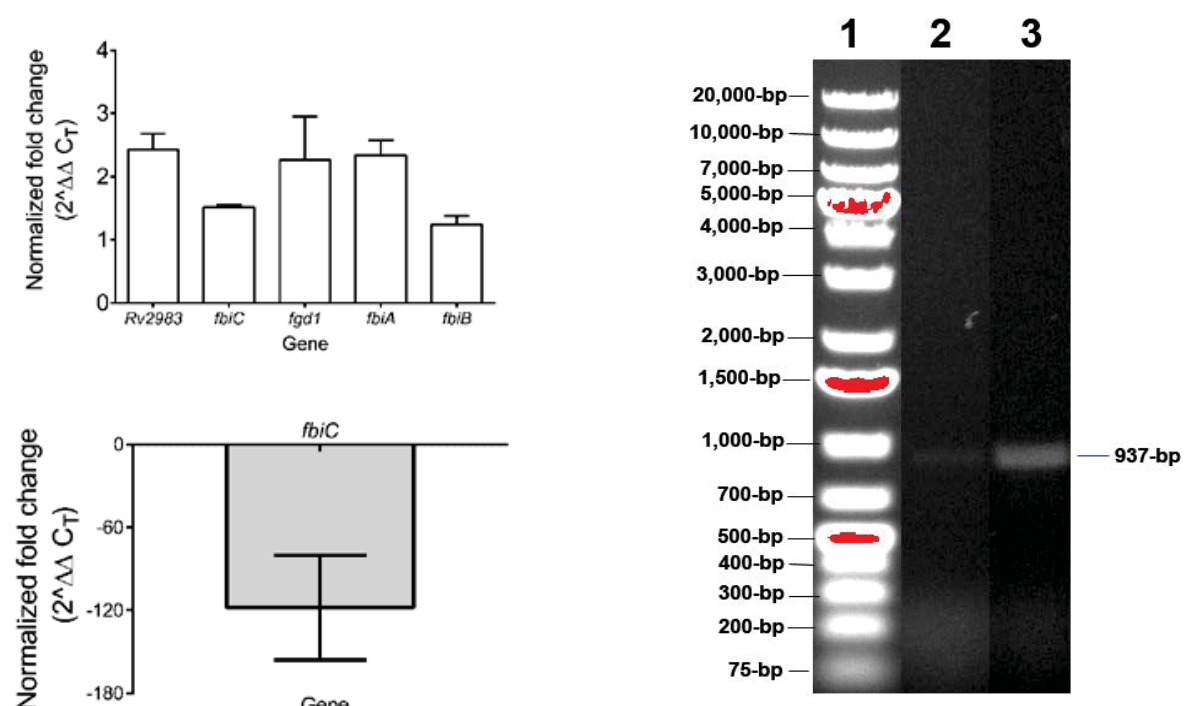

Figure S2. Expression of Rv2983 and other genes involved in nitroimidazole activation. A. Expression of Rv2983 and other genes involved in nitroimidazole activation is higher in the Rv2983 mutant B101 relative to the wild-type H37Rv after 4 days of incubation in 7H9 broth; B. fbiC expression is dramatically lower in the fbiC mutant KA026 relative to the wild-type after 2 days of incubation in $7 \mathrm{H} 9$ broth; $\mathrm{C}$. A faint band representing the 937-bp fbiC DNA fragment is evident in the sample from the KA026 mutant (lane 2) relative to that in H37Rv (lane 3). Lane 1 is the 1-kb DNA marker. 
A.

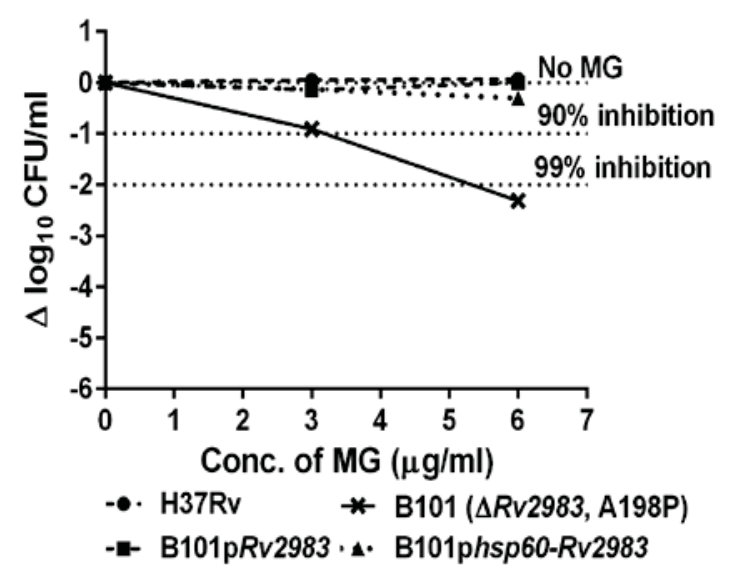

B.

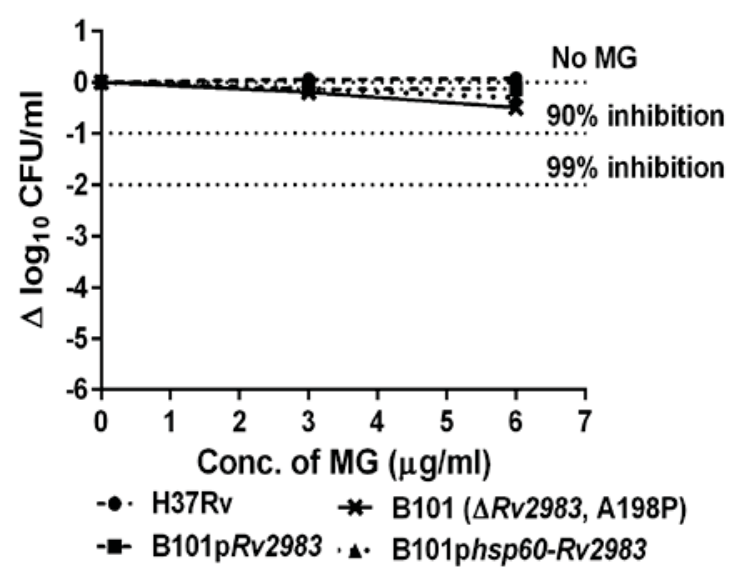

Figure S3. Complementation of the B101 mutant with wild-type RV2983 restores tolerance to MG. The proportional recovery of the mutant on $6 \mu \mathrm{g} / \mathrm{ml}$ of MG increases with the volume of culture plated and the duration of incubation: 28-day incubation of $500 \mu \mathrm{l}(\mathrm{A})$ aliquots/plate; 35-day incubation of $500 \mu \mathrm{l}(\mathrm{B})$ aliquots/plate. 
bioRxiv preprint doi: https://doi.org/10.1101/457754; this version posted October 31, 2018. The copyright holder for this preprint (which was not certified by peer review) is the author/funder. All rights reserved. No reuse allowed without permission. 